\title{
Effectiveness of Acupuncture in Treatment of Simple Obesity in Animal Models: A Systematic Review and Meta-Analysis
}

\author{
Xiao-lin Fan $\mathbb{D}^{1},{ }^{1,2}$ Mei-ling Yu, ${ }^{1}$ Shu-Ping Fu $\mathbb{D}^{\mathbb{D}},{ }^{1,3}$ Yi Zhuang $\mathbb{D},{ }^{1}$ and Sheng-feng Lu $\mathbb{D}^{1,3}$ \\ ${ }^{1}$ The No. 2 Clinical Medicine College, Nanjing University of Chinese Medicine, Nanjing 210023, China \\ ${ }^{2}$ Nanjing Vocational Health College, Nanjing 210023, China \\ ${ }^{3}$ Key Laboratory of Acupuncture and Medicine Research of Ministry of Education, Nanjing University of Chinese Medicine, \\ Nanjing 210023, China
}

Correspondence should be addressed to Sheng-feng Lu; lushengfeng@njucm.edu.cn

Received 18 December 2018; Revised 16 April 2019; Accepted 21 May 2019; Published 14 July 2019

Academic Editor: I-Min Liu

Copyright (C) 2019 Xiao-lin Fan et al. This is an open access article distributed under the Creative Commons Attribution License, which permits unrestricted use, distribution, and reproduction in any medium, provided the original work is properly cited.

Background. Simple obesity has become a global risk to health of human beings. Acupuncture, as one of traditional Chinese medicine therapies, has been widely used in obesity treatment in recent years. However, the individual heterogeneity which makes acupuncture's efficiency unstable leads to some controversy. So more evidence-based results are necessary to judge the effectiveness of acupuncture in treatment of simple obesity. Compared with clinical trials, animal experiments are controllable, and the underlying mechanism is more likely to be explored. Last but not the least, more and more experimental studies on acupuncture for animal obesity have been published. Therefore, we conducted the systematic review and meta-analysis to evaluate the effectiveness of acupuncture in treating simple obesity in animal experiments. Methods. Randomized Controlled Trials (RCTs) on acupuncture for simple obesity animal models were searched from six databases: PubMed, MEDLINE, CNKI, VIP, WanFang Date, and CMB from inception to February 2017 and updated on April 12, 2019. RevMan 5.3 software was used for meta-analysis. Treatment effects were summarized as relative risk (RR) and Standard mean difference (SMD) with 95\% of confidence interval (CI). Results. A total of 108 trials involving 5731 rats were included. Meta-analysis showed that acupuncture had better effect on reducing weight (SMD -2.60, 95\%CI: -2.93 to $-2.26, \mathrm{p}<0.00001$ ) and Lee's index (SMD -2.62, 95\%CI:-3.18 to $-2.06, \mathrm{p}<0.00001$ ) compared with control group. However, the methodological quality of included studies was generally poor. Details of blinding were not reported in most studies. In spite of high heterogeneity being observed on the merged data, sensitivity analysis using the leave-one-out approach, subgroup analysis based on different acupuncture techniques, and rat strains and meta-regression all failed to find the sources of heterogeneity. The asymmetric funnel plot suggested publication bias. Besides, adverse events were not reported in any reports. Conclusions. Our review provided positive evidence of acupuncture for simple obesity. Unfortunately, none of the firm conclusions can be drawn due to methodological flaws, high heterogeneity, and publication bias. More high-quality trials are needed in future to get objective conclusions.

\section{Introduction}

Obesity, which is defined as being abnormal or excessive fat accumulation, accompanied with its complications, has been major risk for human health. A recent study that investigated the health effects of overweight and obesity in 195 countries over the past 25 years showed that the prevalence of obesity has doubled in more than 70 countries and has continuously increased in most other countries [1]. Simple obesity, the obesity which links to unhealthy lifestyles while having no other fundamental diseases, accounting for $95 \%$ of the obese, is the major risk factor for a number of chronic diseases, including diabetes, cardiovascular diseases, and cancer [1-3]. In terms of this global public health problem, Western medicine treatment mainly embraces weight loss drugs and surgery. The antiobesity drugs are considered to be taken for those patients whose BMI exceeds 30 , or 27 accompanied with complications, but healthy diet, regular exercise, and behavioral intervention are still effective in curing obesity; for those patients whose BMI exceeds 40 , or 
35 accompanied with complications, surgical treatment is a suitable complementary therapy [4]. It is worth noticing that in antiobesity drugs only orlistat and sibutramine have been approved by the Food and Drug Administration (FDA) [5], and their therapeutic effectiveness and side effects still require long-term follow-up and clinical evaluation [6]. Although surgery is the best treatment from the viewpoint of significant and fast effect, few obese individuals opt for it because of high risk and high cost [7].

In view of Traditional Chinese Medicine (TCM), the primary pathogenesis of simple obesity includes internal stagnation of fluid dampness and meridian blockage with phlegm. The corresponding treatment principle is eliminating dampness and phlegm through invigorating the spleen [8]. As a form of alternative medicine and key component of TCM, acupuncture has been used widely throughout the world and its efficacy is recognized worldwide as shown in "Acupuncture: Review and Analysis of Reports on Controlled Clinical Trial» [9]. Actually, acupuncture has been widely used for treating simple obesity in clinic because of its benefits in various aspects such as lower cost, more remarkable curative ratio, persistent and stable effect, easy operation, and no regaining worries [10-12].

Modern medical research has shown that acupuncture is effective for simple obesity because of its effects on suppressing appetite, reducing food and energy intake, decreasing absorption of nutrients, and improving energy metabolism through regulating the neuroendocrine system, the digestive system, and the material metabolism [13-15]. There are plenty of clinical studies and researches to verify different acupuncture's effectiveness, including routine acupuncture [16], electroacupuncture (EA) [17], auricular point sticking therapy [18], cupping therapy [19], and acupoint catgutembedding therapy [20]. Due to ethical considerations, clinical trials are unable to obtain detailed information of the acupuncture for obesity. Animal studies could not only limit external factors to get more accurate results but also investigate the underlying mechanism of the experiment. However, few methods can evaluate the clinical applicability of animal experimental studies. The systematic review of animal experiments, which revaluates animal trials through statistical approach to avoid unreasonable decisions is considered as an effective method to explore and improve the guiding value of animal experiments for clinical research [21]. Therefore, we conducted a systematic review and metaanalysis of the effectiveness of acupuncture for losing weight in animal models.

\section{Materials and Methods}

\subsection{Eligibility Criteria}

2.1.1. Types of Studies Included. This systematic review collected randomized controlled trials (RCT) and limited language to Chinese and English.

2.1.2. Types of Subjects. Animal models of simple obesity were included.
2.1.3. Types of Intervention. Acupuncture was the main therapy without restriction on acupuncture types or treatment duration.

2.1.4. Types of Outcomes. Weight and Lee's index (tested at the end of treatment) were chosen as the primary outcomes to evaluate the effectiveness of acupuncture. Weight and Lee's index are classic tests to reflect degree of animal obesity and have been widely applied to animals' experiments.

2.2. Search Strategy. The following six electronic databases were searched from inception to February 2017 and updated on April 12, 2019: PubMed, Medline, the China National Knowledge Infrastructure (CNKI), Chinese Science and Technology Periodical Database (VIP), WanFang data Information Site, and Chinese Biology Medicine Disc (CBMdisc). All searches were restricted on animal experiments but without limits to publications. The search terms utilized consisted of two groups with the medical subject headings: intervention (acupuncture and other related terms) and object (simple obesity and other related terms) in both Chinese and English databases.

2.3. Studies Selection. Only those studies which assessed the use of acupuncture for simple obesity in animal models were included in this review. Two authors (XLF and MLY) independently screened literatures from databases and listed the titles according to the above eligibility criteria. Two reviewers (XLF and MLY) independently carried out the next studies selection, discussed their differences, assessed the risk of bias, and extracted data of the selected articles. Any inconsistency in this process was discussed with the third person (SFL)

2.4. Data Extraction. Two authors (XLF and MLY) independently conducted data extraction and the controversy was resolved by discussion. The last data was compared from the following four aspects: (1) basic information, including the name of the first author, the year of publication, and model of simple obesity; (2) baseline characteristics of animal models, including the species, number, and weight; (3) aspects of treatment, including duration, frequency, course of treatment, and details of acupuncture; and (4) main outcomes (weight and Lee's index) and intergroup difference.

2.5. Quality Assessment. Two authors (XLF and MLY) used a ten-item scale to evaluate the methodological quality of the included studies. Ten items included peer-reviewed publication, control of temperature, exclusion of stress reaction, random allocation to treatment or control, blind method, sample size calculation, compliance with animal welfare regulations, statement of research support, detailed modeling method, and complete testing data. The study got one quality score as it fulfills each criterion of the item; the final score ranges from zero to ten. The higher the score, the higher the article quality. Any disagreement was discussed with the third author (SFL). 


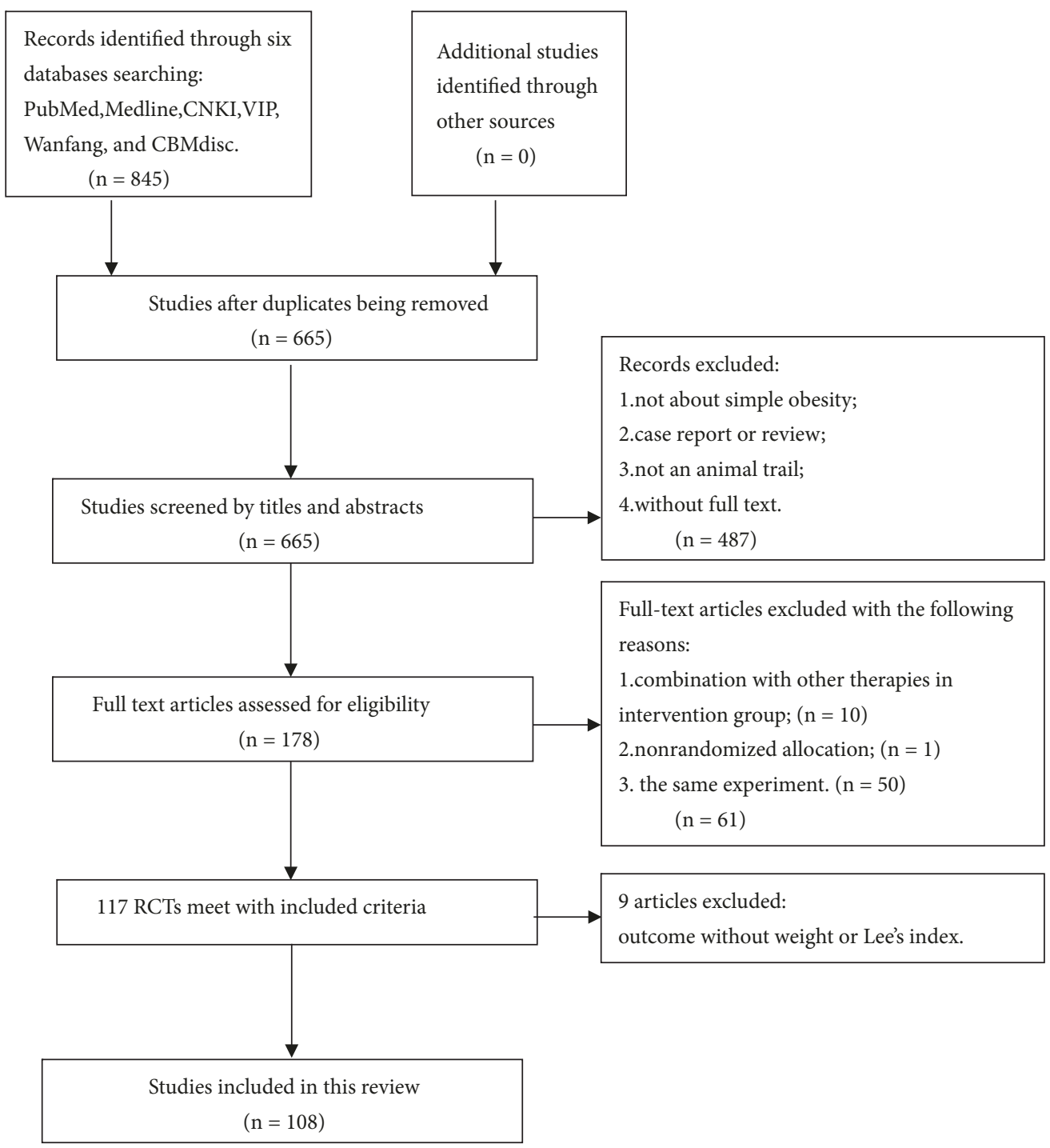

FIGURE 1: Flow diagram of the study selection process for this systematic review and meta-analysis.

2.6. Statistical Analysis. RevMan 5.3 software was used for meta-analysis. Treatment effects were summarized as relative risk (RR) and Standard mean difference (SMD) with a $95 \%$ of confidence interval (CI). The Standard mean difference (SMD) was an evaluation of the combined effect sizes and $P$ values below 0.05 were considered statistically significant. Heterogeneity was calculated with I-squared statistics and $\chi$-squared distribution. Besides, we carried out subgroup analysis based on different acupuncture methods and different animal species. To explore the sources of heterogeneity, we did the subgroup meta-analyses and metaregression. The funnel plot and Egger's and Begg's Test were applied to detect the publication bias.

\section{Results}

3.1. Study Inclusion. We identified 845 potentially relevant articles before February, 2017, from the following six databases: PubMed, Medline, the China National Knowledge Infrastructure (CNKI), Chinese Science and Technology Periodical Database (VIP), WanFang data Information Site, and Chinese Biology Medicine Disc (CBMdisc). After removal of duplicates, there were 665 records remaining. Based on review of titles and abstracts, we ruled 487 papers out because of at least one of the following reasons: (1) not being about simple obesity, (2) case report or review, (3) not an animal trail, and (4) being unable to get full text. After reading all the remaining 178 articles through, 61 studies were excluded for the following considerations and issues: (1) combination with other therapies in control group, (2) nonrandomized allocation, and (3) republication for the same experiment. Among the included 117 studies, 9 studies were ruled out because their main outcome measures consisted of neither weight nor Lee's index. At last, 108 studies were included. The flow diagram of the study selection process is shown in Figure 1. 
3.2. Study Characteristics. 101 studies were written in Chinese and 7 in English. The 7 English studies were all published in international journals and the other 101 Chinese studies all in Chinese Journals. The 108 included studies involve a total of 5731 animals (74 studies with SD rats, 25 studies with Wistar rats, 8 studies with mice, 1 study with guinea pigs, and 3 studies which did not mention specific types of rats). Of all included trials, 13 studies did not provide the specific data of weight. The weight of rats ranges from $40 \mathrm{~g}$ to $120 \mathrm{~g}$ in 63 studies and 130 to $250 \mathrm{~g}$ in 22 studies. The mice in 7 experiments weighed from 9 to $24 \mathrm{~g}$. In terms of gender, the vast majority of trials selected male rats; only 3 trials chose female rats. 12 studies used both male and female rats and 9 studies did not mention the sex. Most of trials (104) induced simple obesity by feeding with high fat diet, 2 trials by sodium glutamate, and 2 trials adopted simple obesity rats directly. Most of the rats, having eliminated stress reaction, were randomly assigned to acupuncture group or control group. Of these included RCTs, 77 studies used electroacupuncture, 16 studies chose handle acupuncture, and 11 studies adopted catgut implantation while 4 studies used auricular acupuncture. No treatment was given to the control group. A total of 56 studies used both weight and Lee's index as main outcome measures. Meanwhile, 48 studies only employed weight and 4 studies only used Lee's index as the outcome measure. Of the four studies with auricular acupuncture, except two studies without specific points, the other two studies chose the following areas on the auricle: Gastric, Small Intestine, Stomach, and Hunger Point. Within all the remaining 104 studies, besides 6 studies which did not give concrete points, we sorted out about 17 acupoints used in experiments: Zusanli (ST 36), Neiting (ST44), Zhongwan (CV 12), Tianshu (ST 25), Sanyinjiao (SP 6), Guanyuan (CV 4), Fenglong (ST 40), Yinlingquan (SP 9), Neiguan (PC 6), Quchi (LI 11), Daheng (SP 15), Zulinqi (GB 41), Weishu (BL 21), Sibai (ST 2), Liangmen (ST 21), Pishu (BL 20), and Guilai (ST 29). Then, we generate a list to calculate the times and frequency of acupoints and meridians used. The leading acupoints Zusanli (ST 36), Zhongwan (CV 12), Neiting (ST 44), Tianshu (ST 25), Sanyinjiao (SP 6), Guanyuan (CV 4), Fenglong (ST 40), and Yinlingquan (SP 9) adopted times were $88,36,34,33,26,41,12$, and 6 , respectively. Correspondingly, the most common used meridian was the Stomach Meridian of Foot-yangming, followed by Conception Vessel and the Spleen Meridian of Foot-Taiyin.

Based on different acupuncture techniques, the frequency and course of treatment were quite different. For catgut implantation therapy, the rats were treated once around every 7 15 days for almost 2 4 cycles. The course of treatment in electroacupuncture, handle acupuncture, and auricular acupuncture therapy were similar, 10 to 30 minutes of retaining needle basically, almost once or twice a day, lasting 8 to 56 days.

Of all the 77 studies that adopted electroacupuncture, only 2 studies ignored the detailed operating parameters. 36 trials, more than half of the studies, used continuous waves with frequency of 1-100 Hz. 14 studies used dispersedense waves with frequency of $2-100 \mathrm{~Hz}$ and 1 study used discontinuous wave with a frequency of $2 \mathrm{~Hz}$. The rest 25 studies provided the frequency of waves but referred no specific wave types. It could be found that continuous wave, $10 \mathrm{~Hz}$ of frequency, was the most commonly used stimulus parameter of electroacupuncture for obesity. Even reinforcing reducing by twirling was the commonly adopted manipulating technique in manual acupuncture groups. The basic characteristics of the 108 studies are shown in Table 1.

3.3. Study Quality. The study quality checklist items score ranged from 3 to 8 out of total 10 points, which includes such items as peer-reviewed publication, control of temperature, random allocation to treatment or control, blinding, stress reaction, sample size calculation, detailed molding methods, complete outcome data, compliance with animal welfare regulations, and statement of potential conflict of interests or foundation items. Of the 108 included studies, one study got 3 points; twelve studies got 4 points; forty-two studies got 5 points; thirty-five studies got 6 points; sixteen studies got 7 points and one study got 8 points. Seventy-seven studies were rigorously peer-reviewed before publication. In fifty trials, temperature was controlled while one study did not describe the explicit temperature. Randomization was performed in all trials while only one study reported blinding. Two studies failed to rule out stress reaction. No trial reported sample size calculation. Ninety-nine studies described detailed methods of establishing the animal model including process and time consuming. One hundred and two studies gave the complete results and analysis of those experiments. Forty-two studies mentioned their foundation items (Table 2).

3.4. Effectiveness. Eighty-seven studies showed significant beneficial effects of acupuncture for losing weight compared with control group $(n=1695$, SMD -2.60, 95\%CI: $-2.93 \sim-2.26$, $\mathrm{p}<0.00001$; heterogeneity $\mathrm{X}^{2}=507.17, \mathrm{p}<0.00001, \mathrm{I}^{2}=83 \%$, Figure 2); although the left seventeen studies also reported effects of acupuncture on losing weight in comparison with control group, they failed to be incorporated into metaanalysis because of lacking specific data to produce a data graph $(\mathrm{p}<0.05$ or $\mathrm{p}<0.01)$.

In sixty of included publications, Lee's index was reported after acupuncture, but ten of them have no statistical analysis due to short of concrete data $(\mathrm{p}<0.05$ or $\mathrm{p}<0.01)$; the other forty-eight publications reported statistically significant effect of acupuncture on decreasing Lee's index compared with the control group ( $\mathrm{n}=1075$, SMD -2.62, 95\%CI: -3.18 -2.06, $\mathrm{p}<0.00001$; heterogeneity $\mathrm{X}^{2}=487.62$, $\mathrm{p}<0.00001, \mathrm{I}^{2}=90 \%$, Figure 3 ).

However, large heterogeneity on the merged data was hard to ignore (heterogeneity of merged weight: $\mathrm{I}^{2}=83 \%$, $\mathrm{p}<0.00001$; heterogeneity of merged Lee's index: $\mathrm{I}^{2}=90 \%$, $\mathrm{p}<0.00001)$.

3.5. Sensitivity Analysis. We made sensitivity analysis on the merged data, which did not decrease heterogeneity.

3.6. Subgroup Analysis. Since different acupuncture techniques and rat strains were employed by the included studies, 


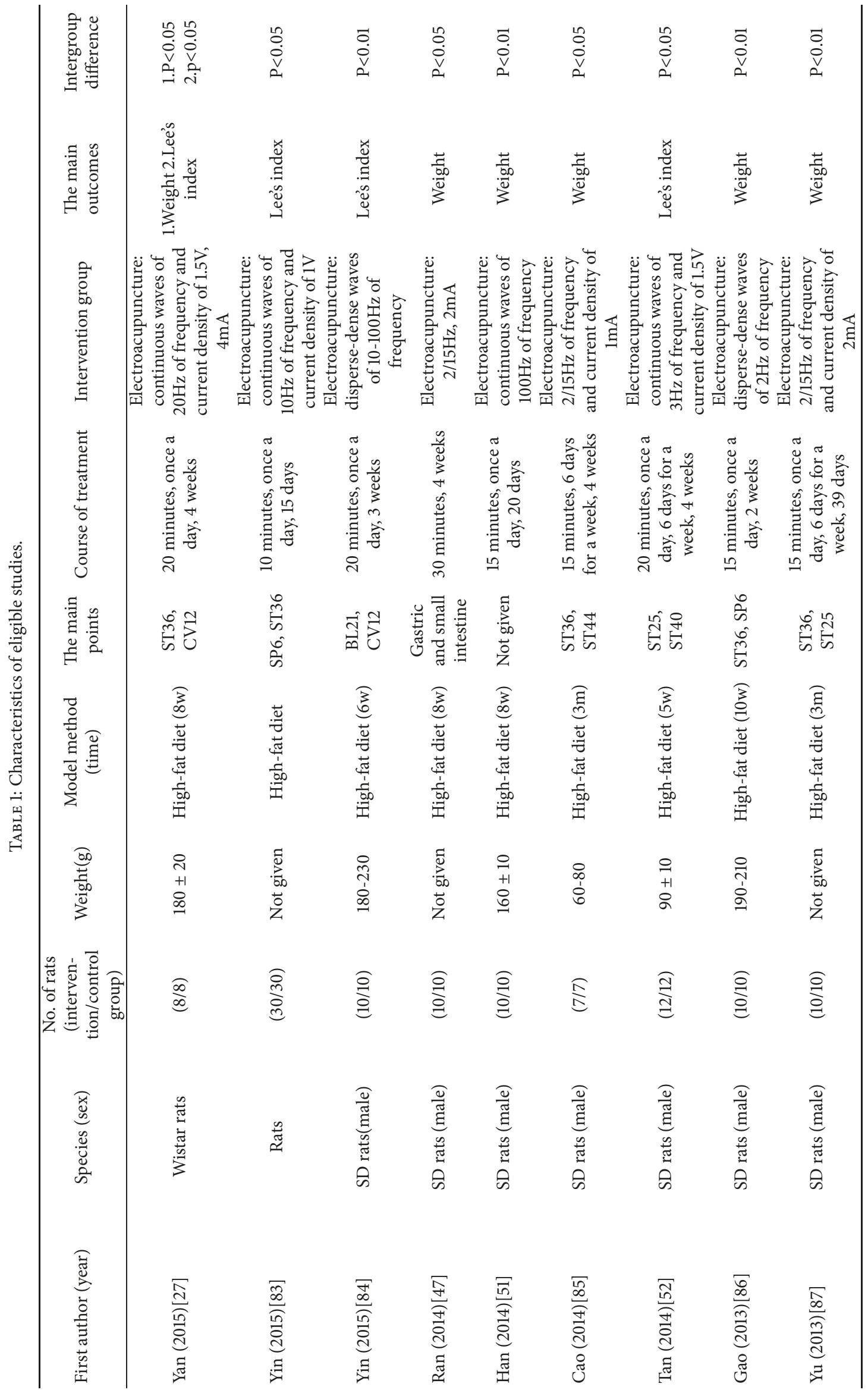




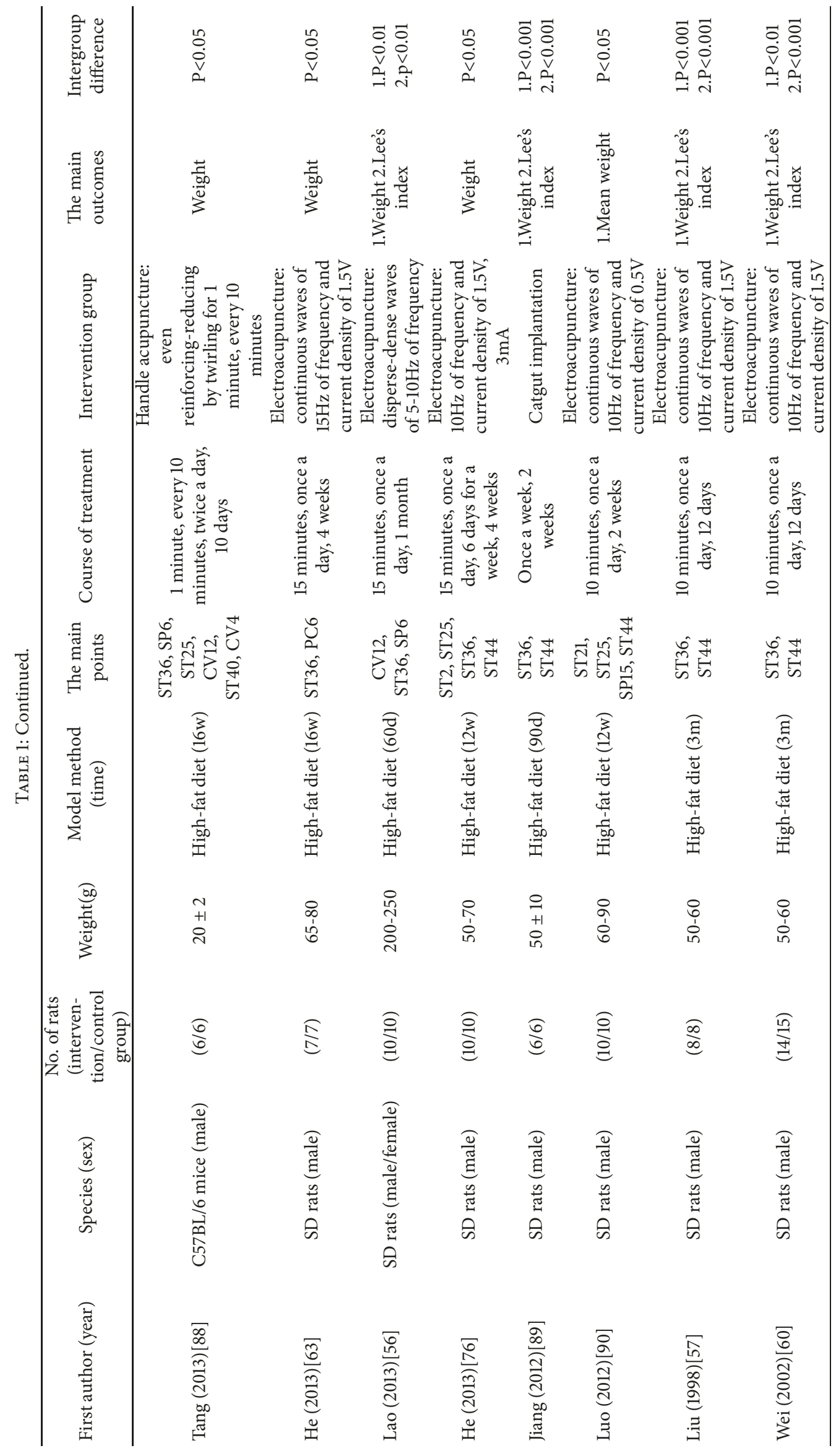




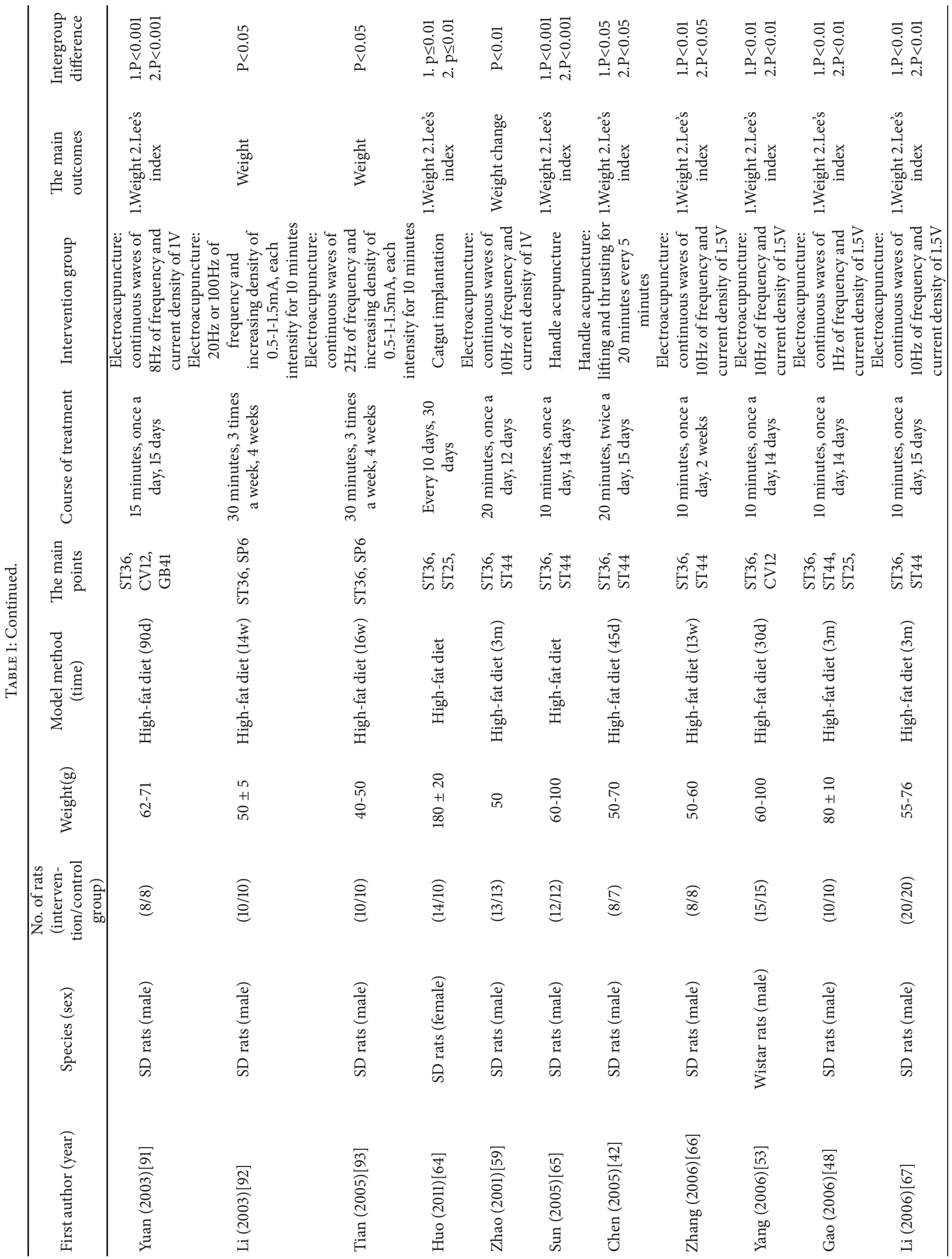




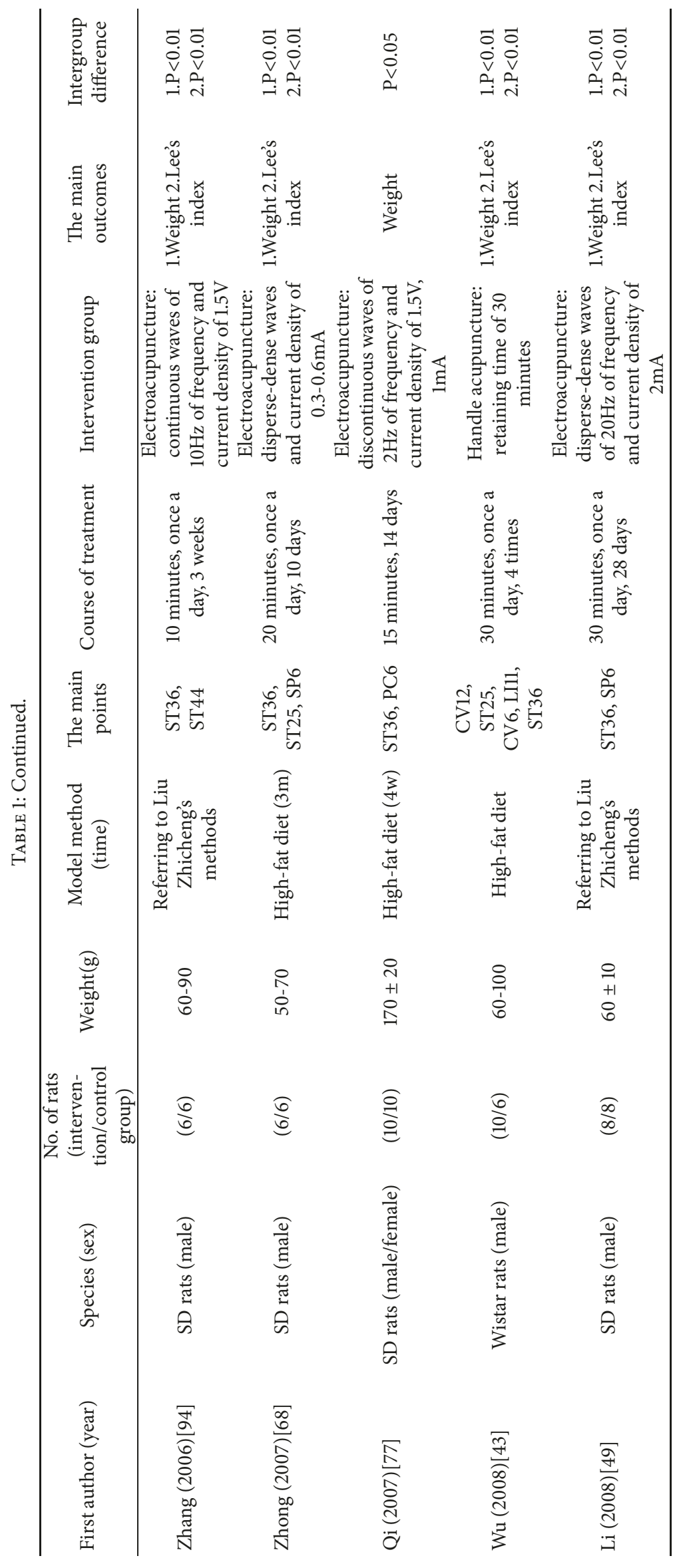




\begin{tabular}{|c|c|c|c|c|c|}
\hline 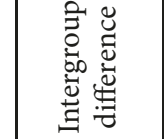 & 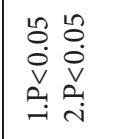 & 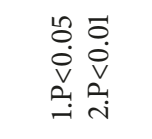 & $\begin{array}{l}\vec{b} \\
\dot{\vec{v}} \\
\text { ni }\end{array}$ & 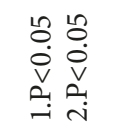 & $\begin{array}{l}\vec{D} \\
\dot{\vec{v}} \\
\stackrel{a}{1}\end{array}$ \\
\hline 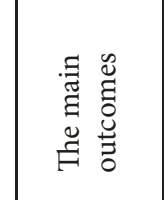 & 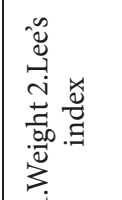 & 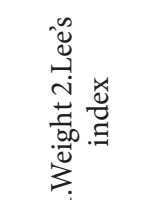 & 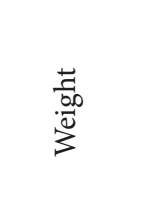 & 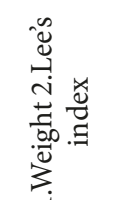 & 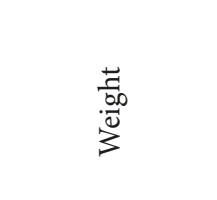 \\
\hline 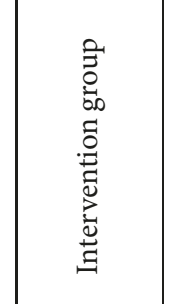 & 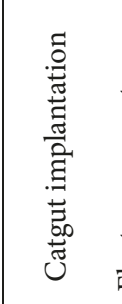 & 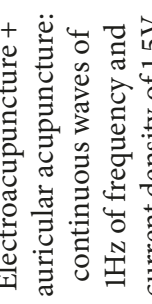 & 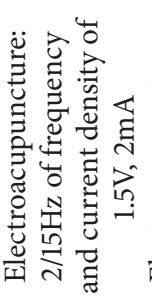 & 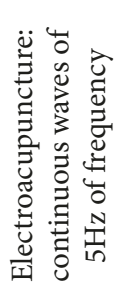 & 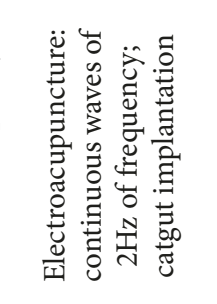 \\
\hline 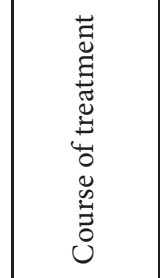 & 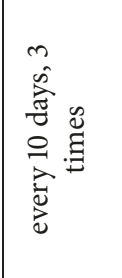 & 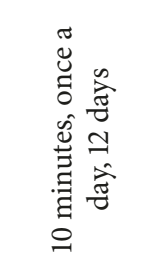 & 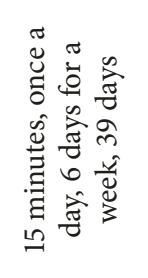 & 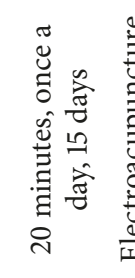 & 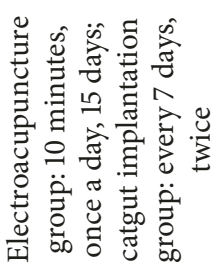 \\
\hline 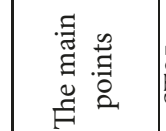 & 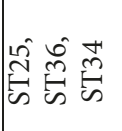 & 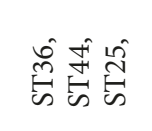 & 总葛 & 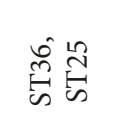 & 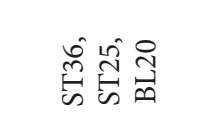 \\
\hline 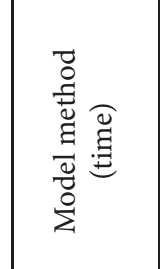 & 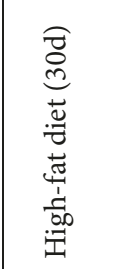 & 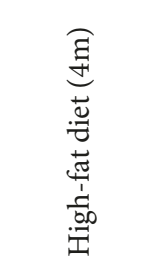 & 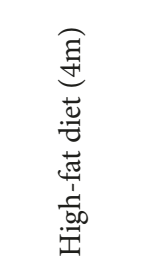 & 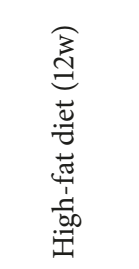 & 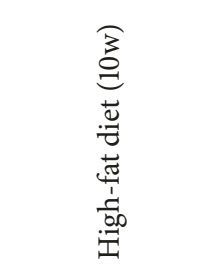 \\
\hline 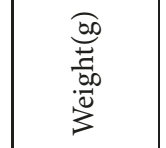 & $\begin{array}{l}\text { in } \\
+1 \\
8 \\
0\end{array}$ & $\begin{array}{l}\stackrel{0}{0} \\
\stackrel{1}{R}\end{array}$ & $\begin{array}{l}0 \\
0 \\
i \\
\text { in }\end{array}$ & $\begin{array}{l}\text { in } \\
+1 \\
10 \\
i n\end{array}$ & 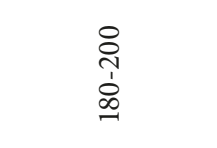 \\
\hline 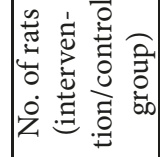 & $\frac{\widehat{\infty}}{\infty}$ & $\frac{\widehat{\infty}}{\infty}$ & 量 & बे & త్ \\
\hline 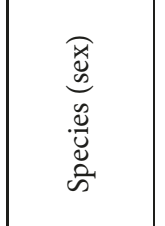 & 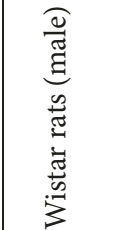 & 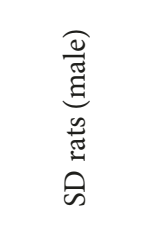 & 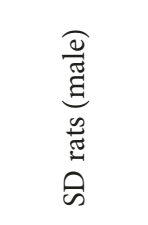 & 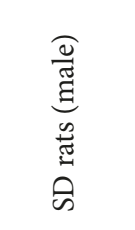 & 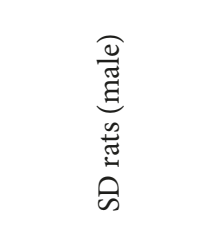 \\
\hline 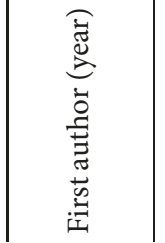 & 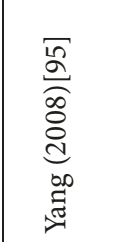 & 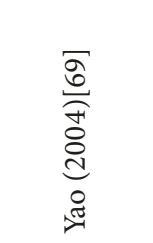 & 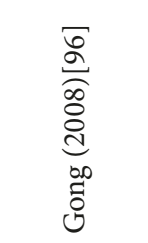 & 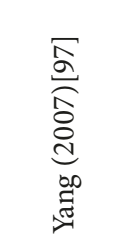 & 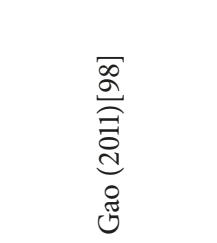 \\
\hline
\end{tabular}




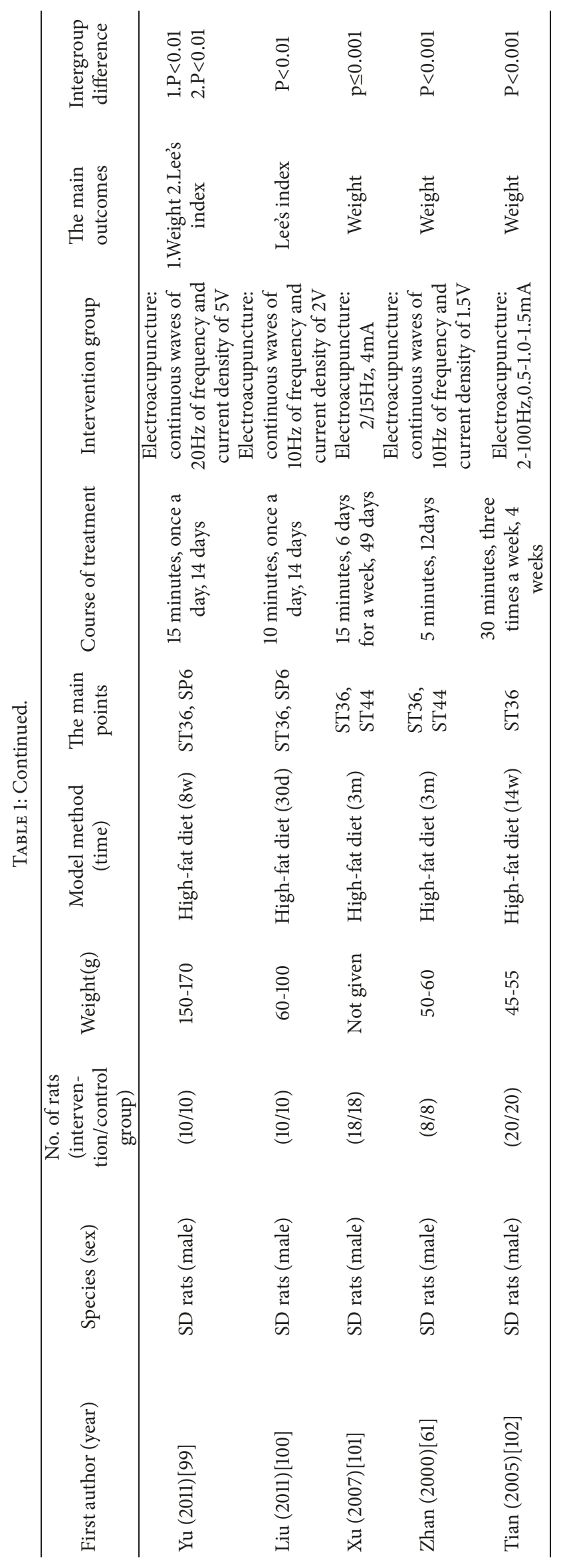




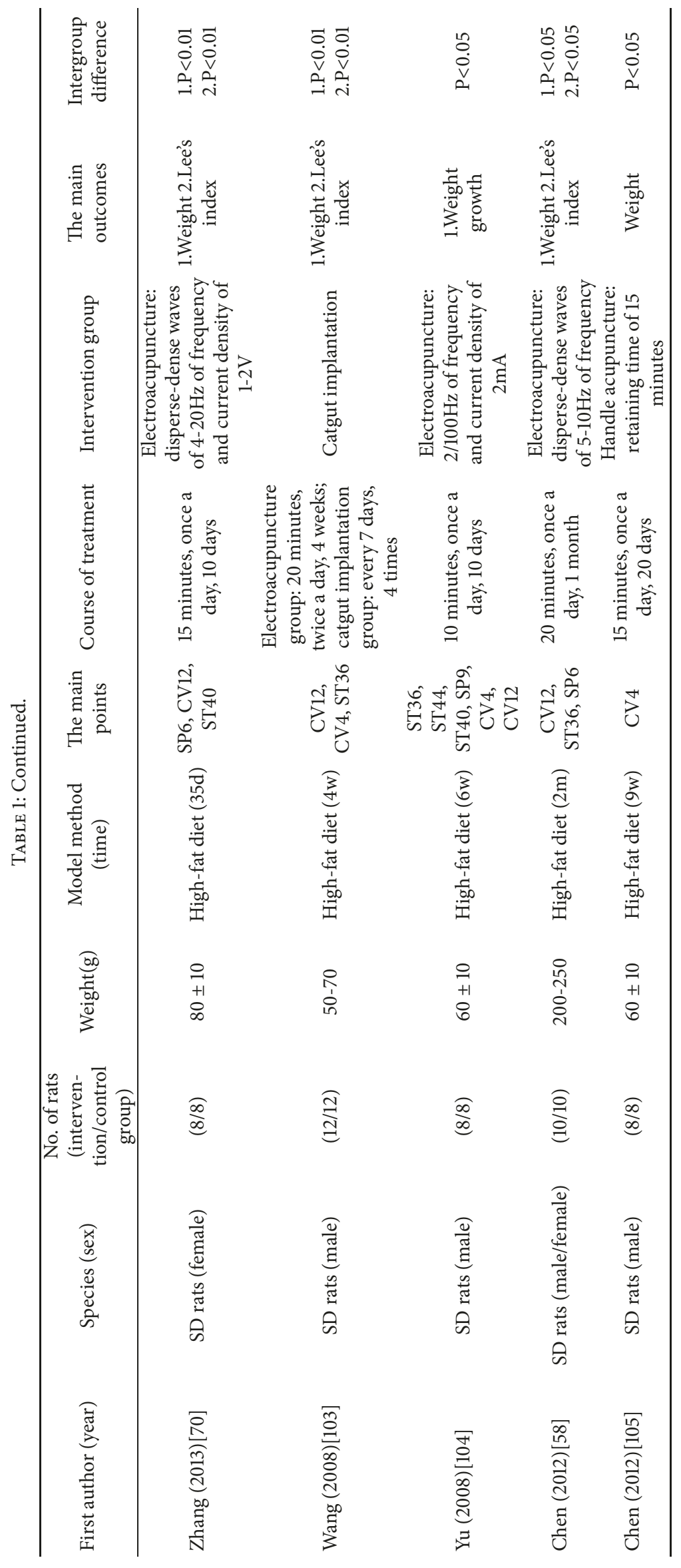




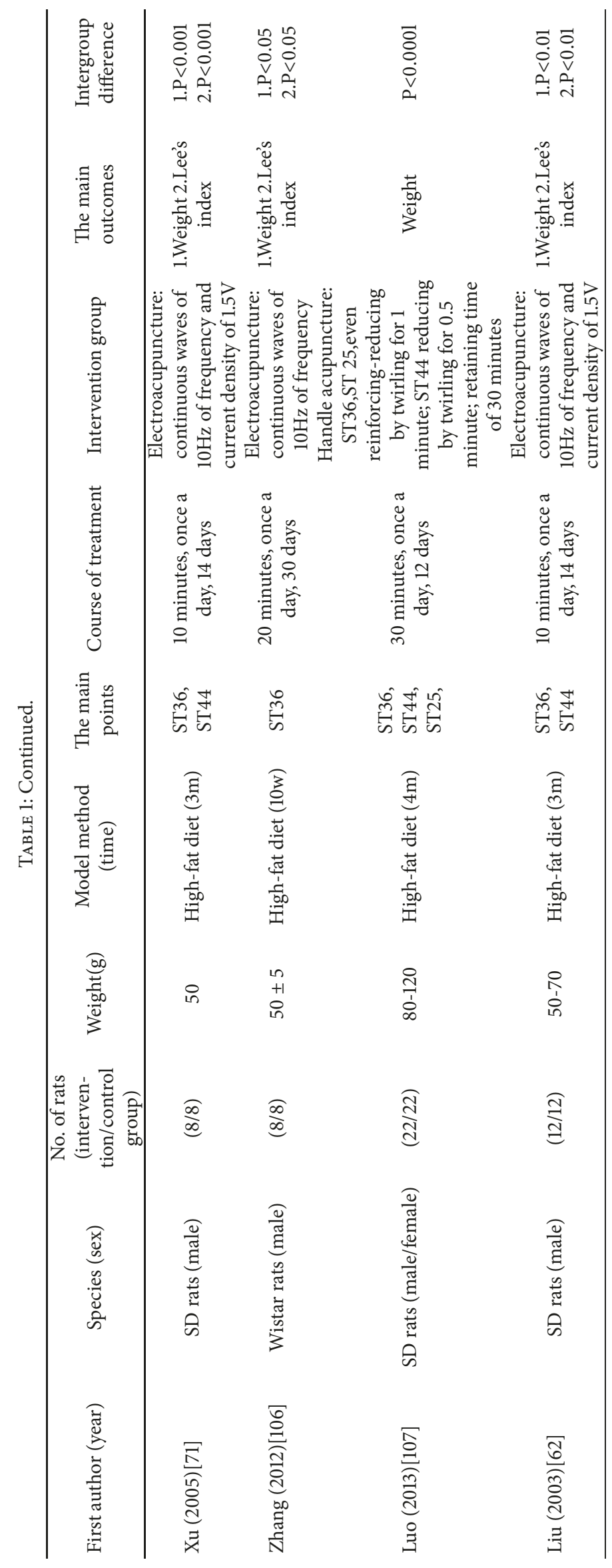




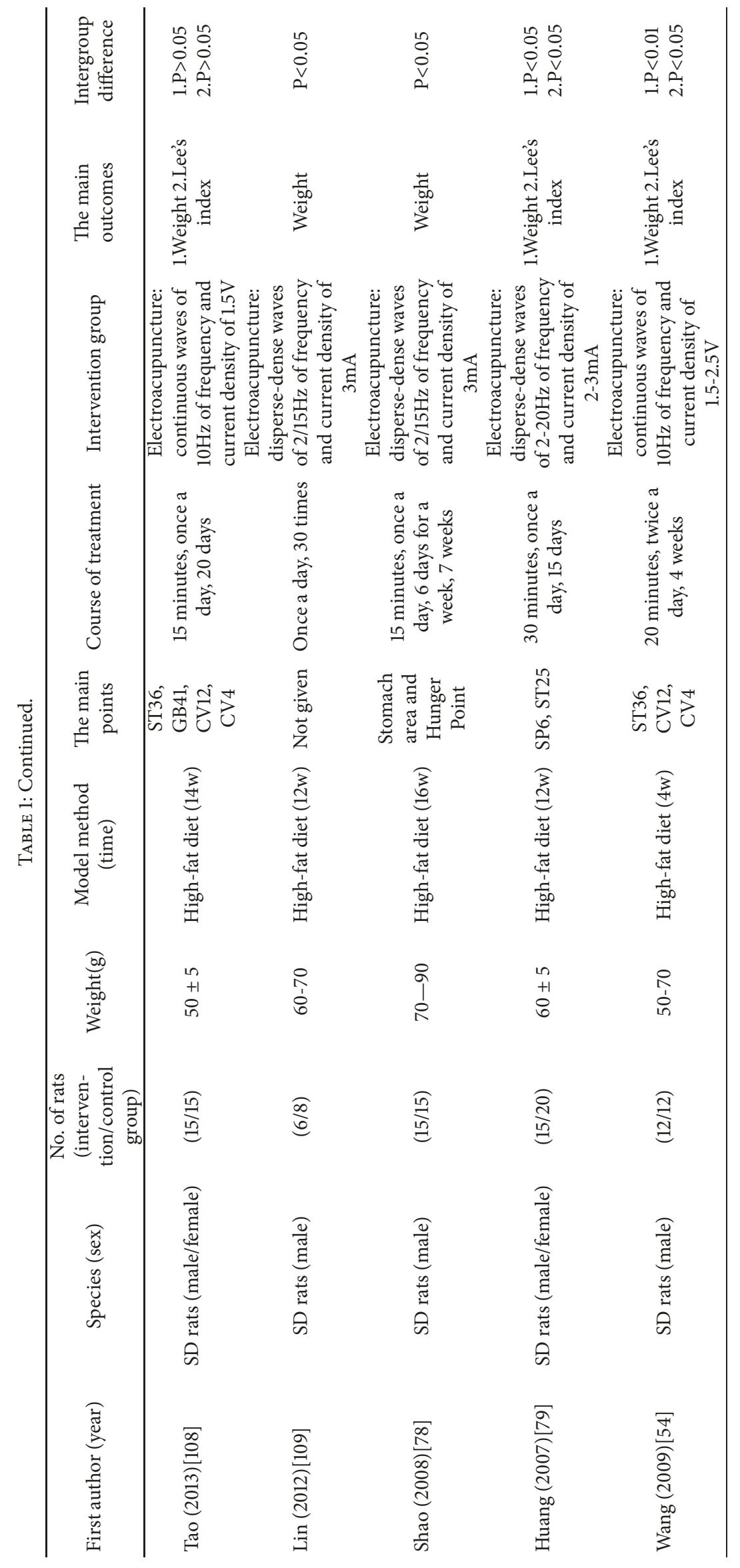




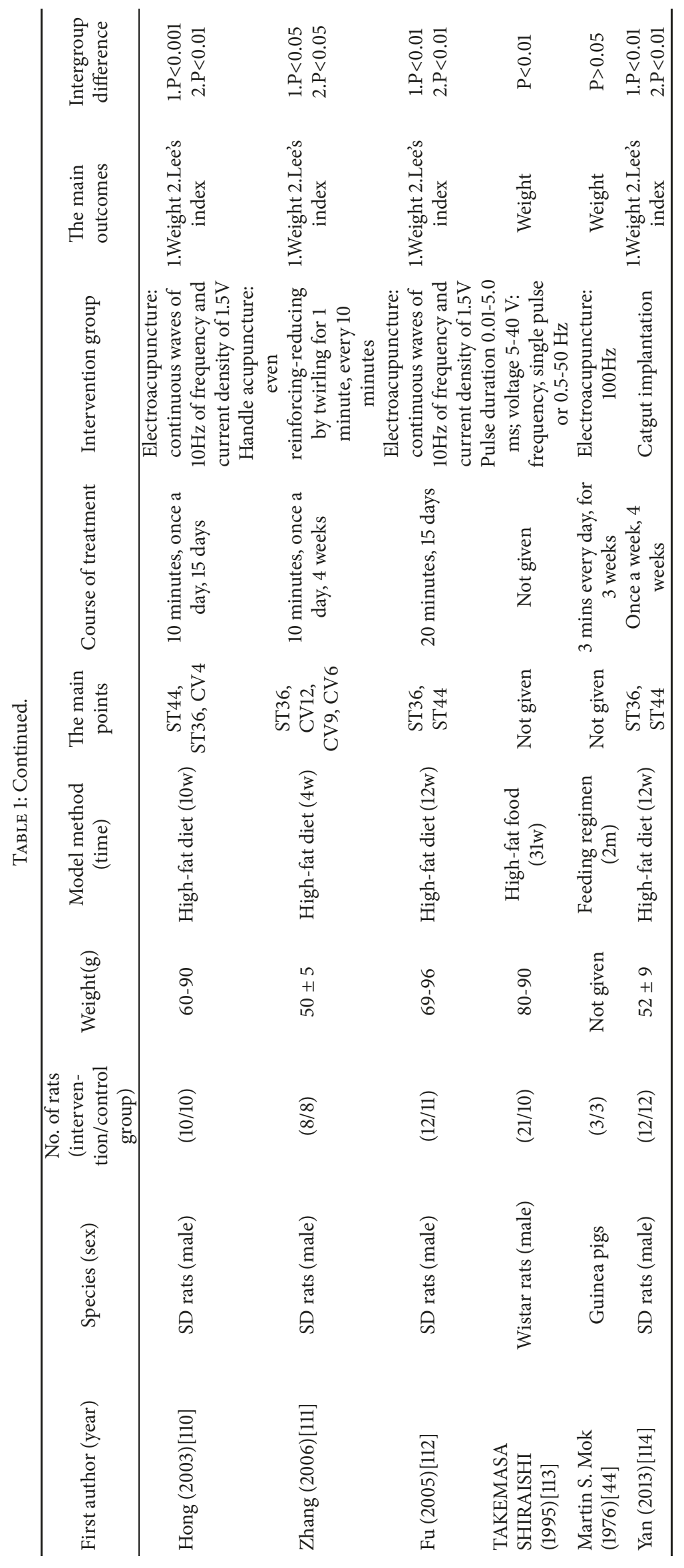




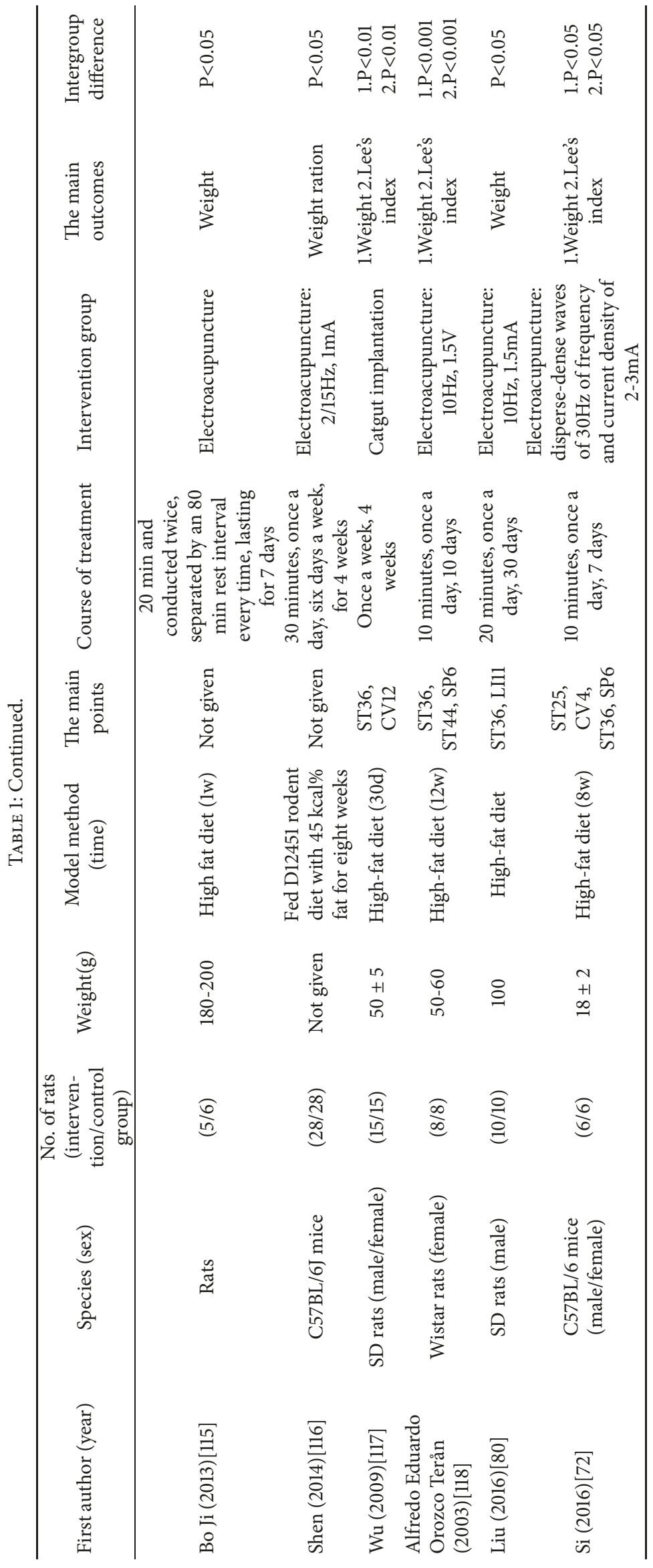




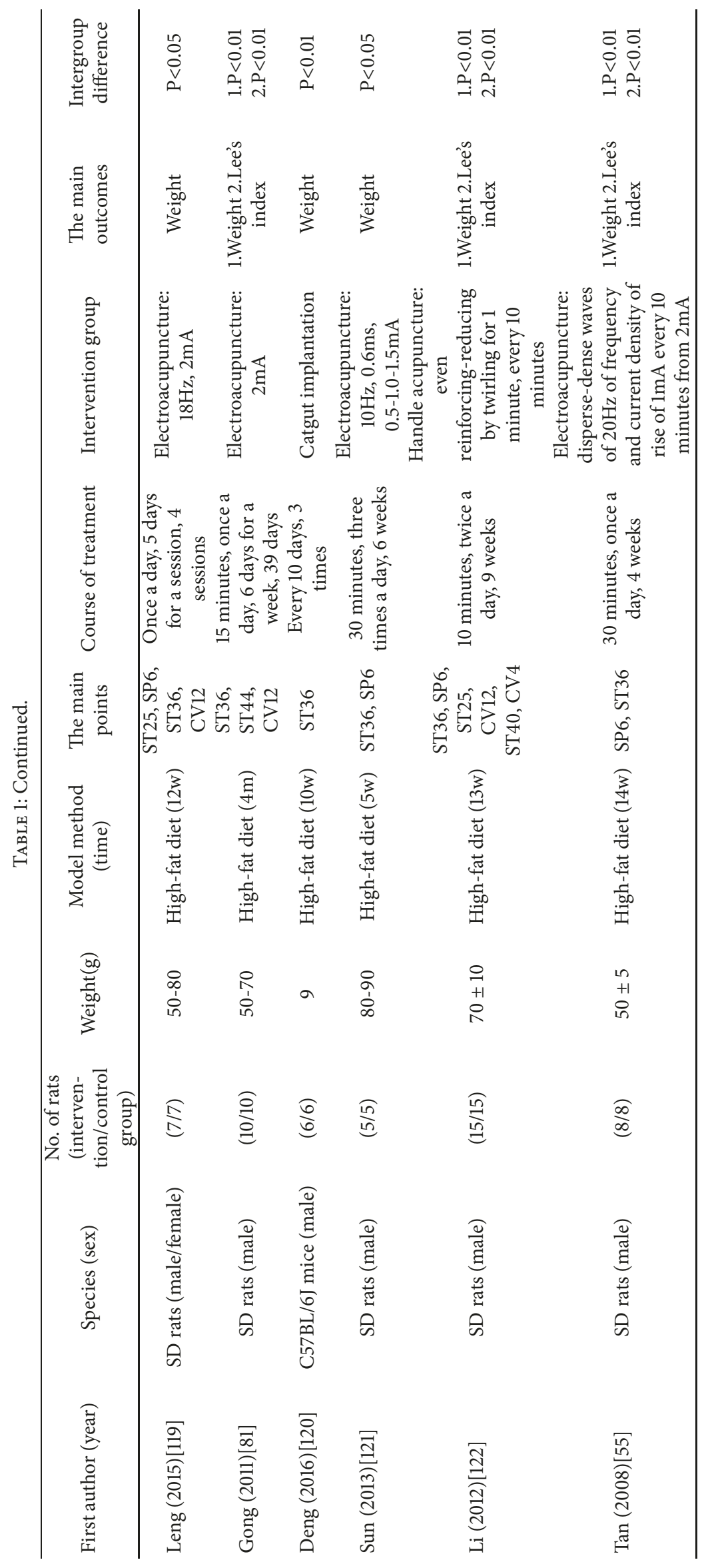




\begin{tabular}{|c|c|c|c|c|c|}
\hline 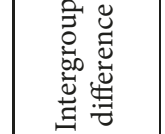 & 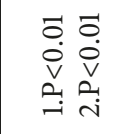 & $\begin{array}{l}\overrightarrow{0} \\
\dot{0} \\
0 \\
0\end{array}$ & 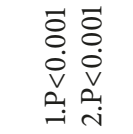 & 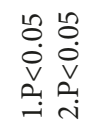 & 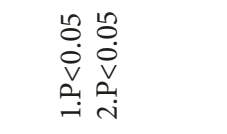 \\
\hline 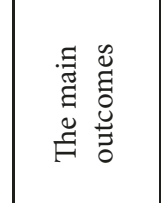 & 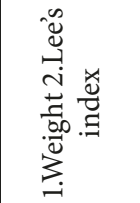 & 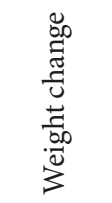 & 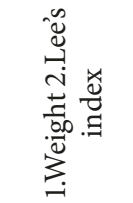 & 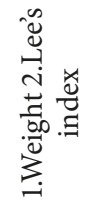 & 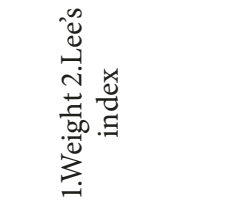 \\
\hline 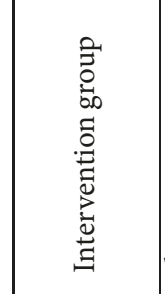 & 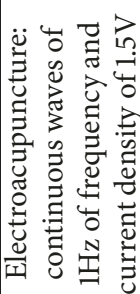 & 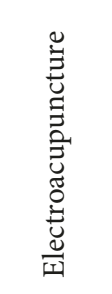 & \multicolumn{2}{|c|}{ 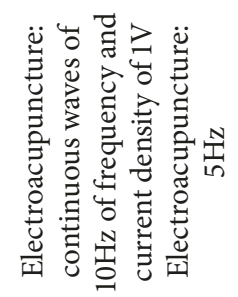 } & 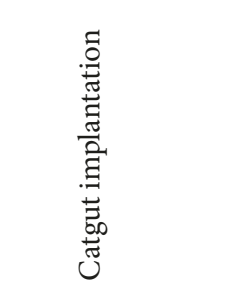 \\
\hline 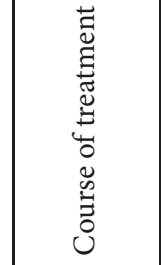 & 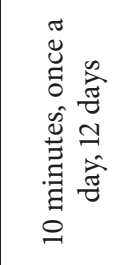 & 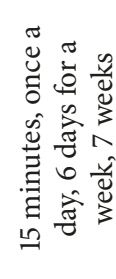 & 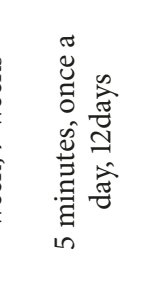 & \multicolumn{2}{|c|}{ 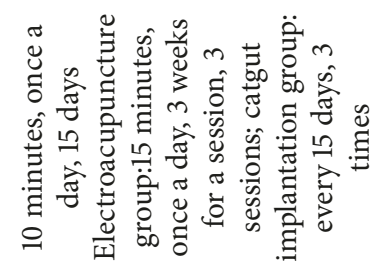 } \\
\hline 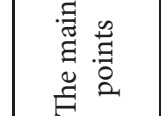 & 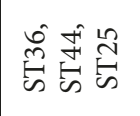 & 总茊 & 兽薦 & 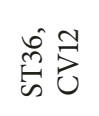 & 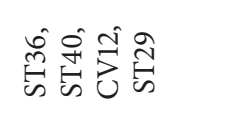 \\
\hline 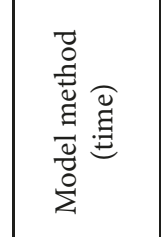 & 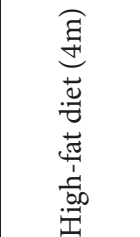 & 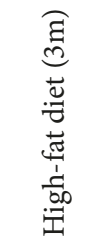 & 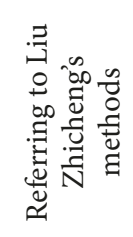 & 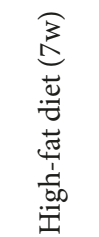 & 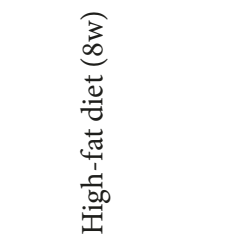 \\
\hline $\begin{array}{l}300 \\
\frac{30}{0.0} \\
.00 \\
3 \\
3\end{array}$ & $\begin{array}{l}\infty \\
\infty \\
0 \\
1\end{array}$ & 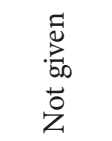 & $\begin{array}{l}8 \\
0 \\
0 \\
i n\end{array}$ & $\begin{array}{l}\text { R } \\
\text { ì } \\
\text { in }\end{array}$ & $\begin{array}{l}0 \\
i \\
+1 \\
0 \\
\infty \\
0\end{array}$ \\
\hline 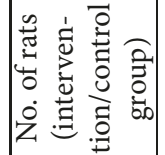 & $\frac{\infty}{\infty}$ & $\stackrel{0}{\stackrel{0}{O}}$ & 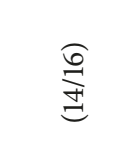 & $\frac{\sqrt{n}}{6}$ & $\frac{\widehat{\infty}}{\infty}$ \\
\hline 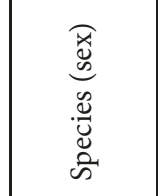 & 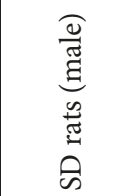 & 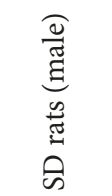 & 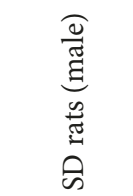 & 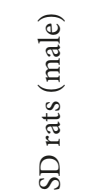 & 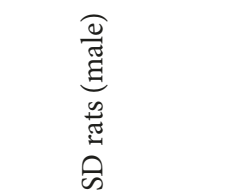 \\
\hline 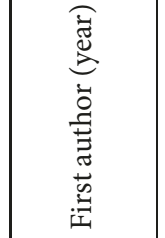 & 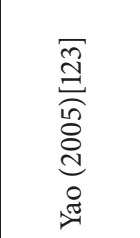 & 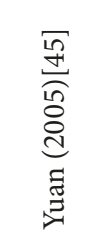 & 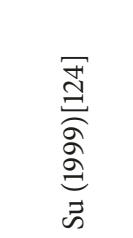 & 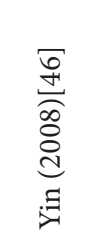 & 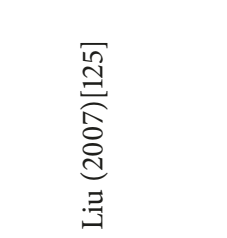 \\
\hline
\end{tabular}




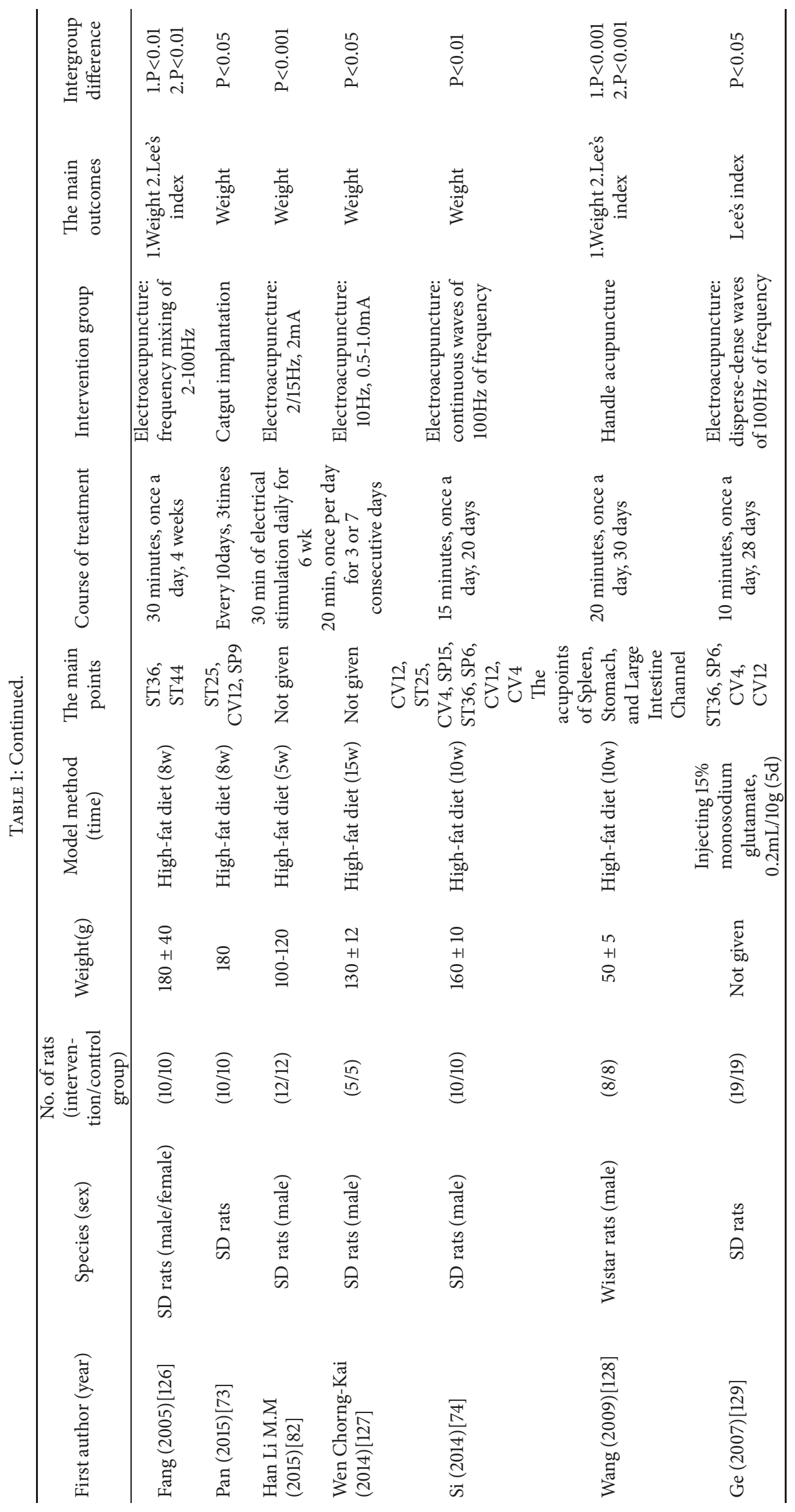




\begin{tabular}{|c|c|c|c|c|c|c|}
\hline 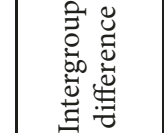 & 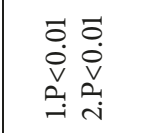 & 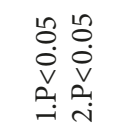 & 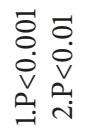 & 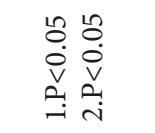 & $\begin{array}{l}u \\
o \\
\dot{v} \\
v \\
v_{1}\end{array}$ & 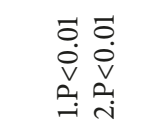 \\
\hline 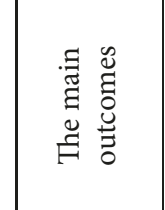 & 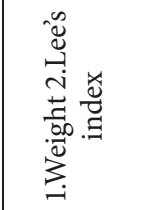 & 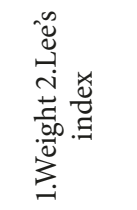 & 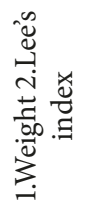 & 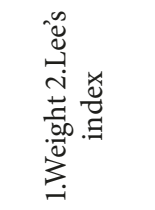 & $\begin{array}{l}\frac{7}{5} \\
\frac{.00}{00} \\
3 \\
3\end{array}$ & 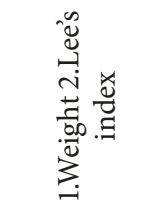 \\
\hline 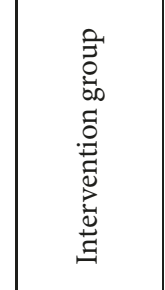 & 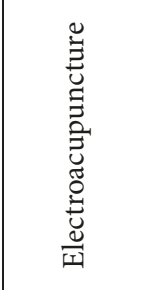 & 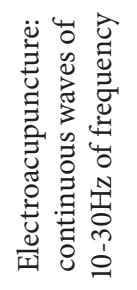 & 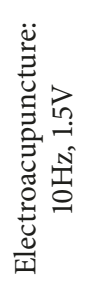 & 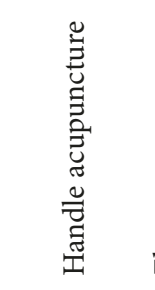 & 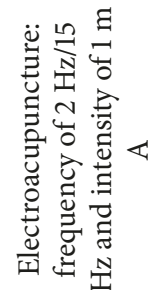 & 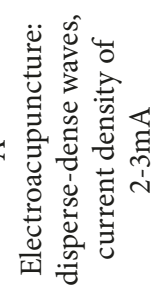 \\
\hline 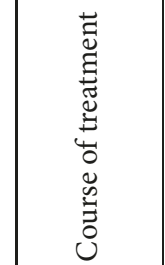 & 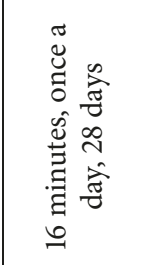 & 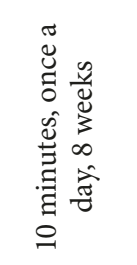 & 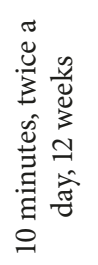 & 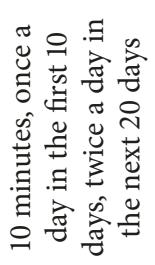 & 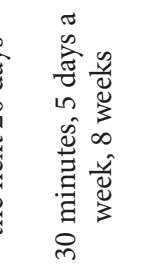 & 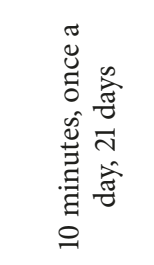 \\
\hline 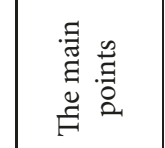 & 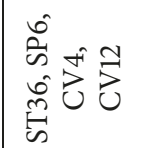 & 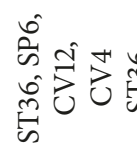 & 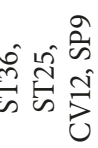 & $\begin{array}{l}\stackrel{\omega}{\sim} \\
\stackrel{n}{5}\end{array}$ & 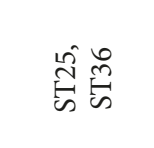 & 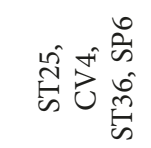 \\
\hline 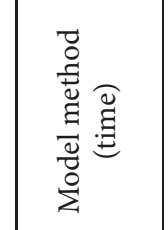 & 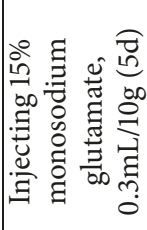 & 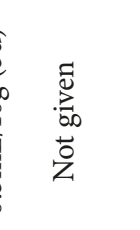 & 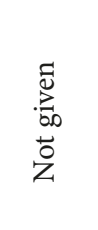 & 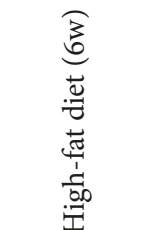 & 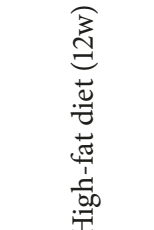 & 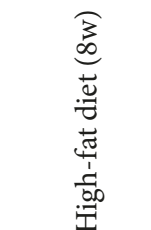 \\
\hline $\begin{array}{l}\widehat{000} \\
\frac{5}{0.0} \\
.00 \\
30\end{array}$ & 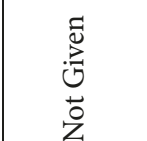 & 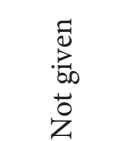 & 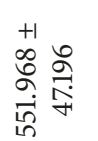 & $\underset{\sim}{\infty}$ & 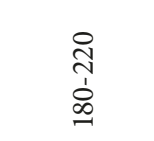 & $\begin{array}{l}N \\
+1 \\
\infty \\
\simeq\end{array}$ \\
\hline 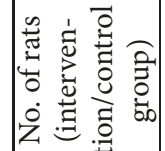 & 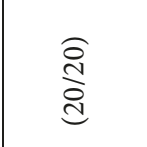 & $\frac{6}{6}$ & $\frac{\infty}{\infty}$ & $\stackrel{\stackrel{\mathrm{O}}{O}}{\mathrm{O}}$ & $\frac{\infty}{\infty}$ & $\stackrel{\varrho}{\stackrel{9}{O}}$ \\
\hline 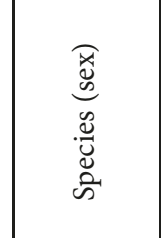 & 营 & 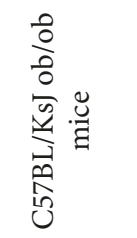 & 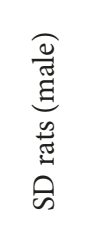 & 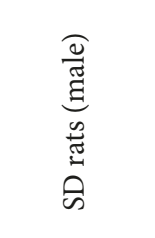 & 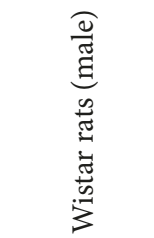 & 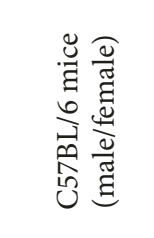 \\
\hline 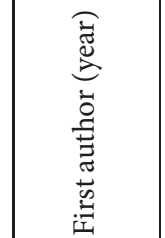 & 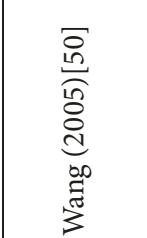 & 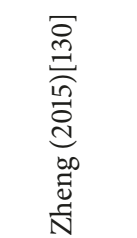 & 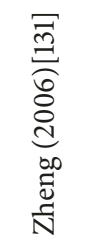 & 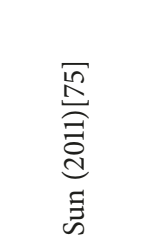 & 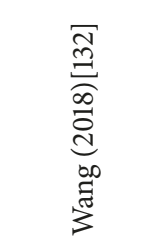 & 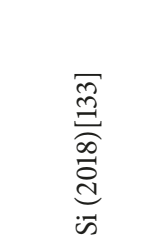 \\
\hline
\end{tabular}




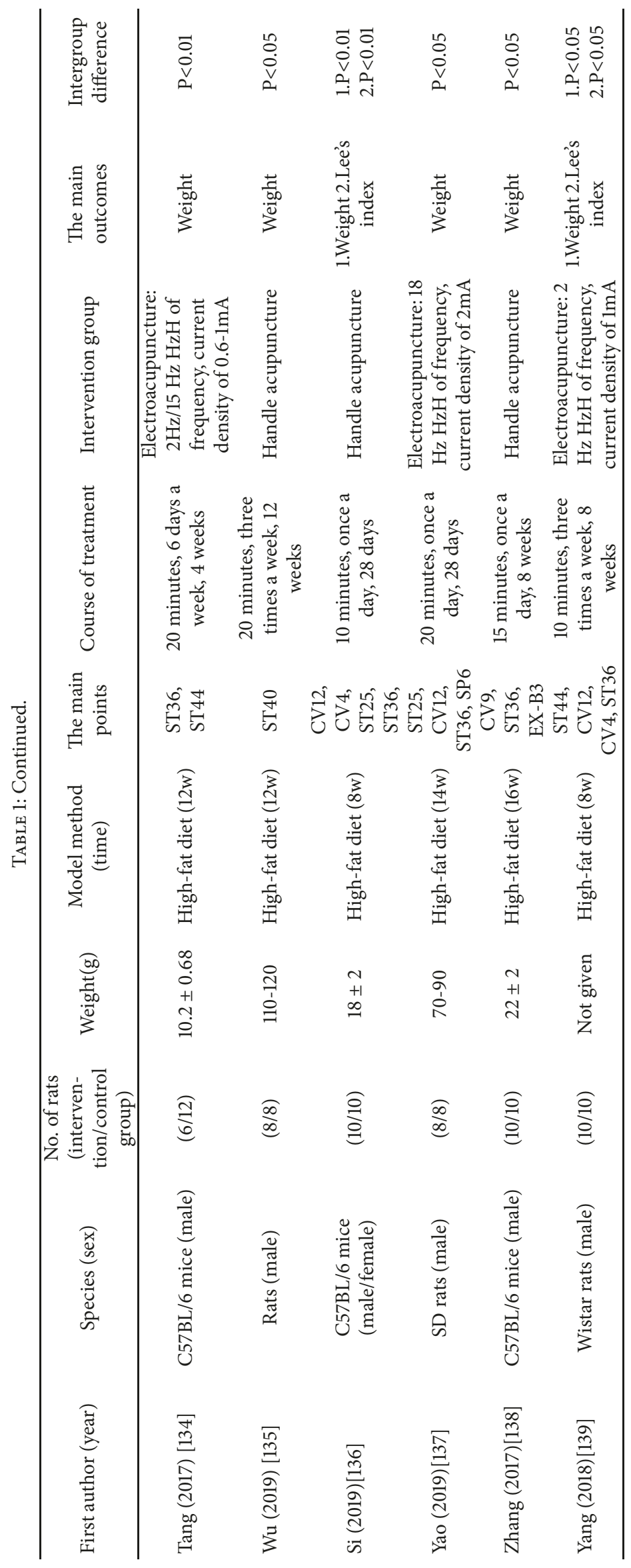




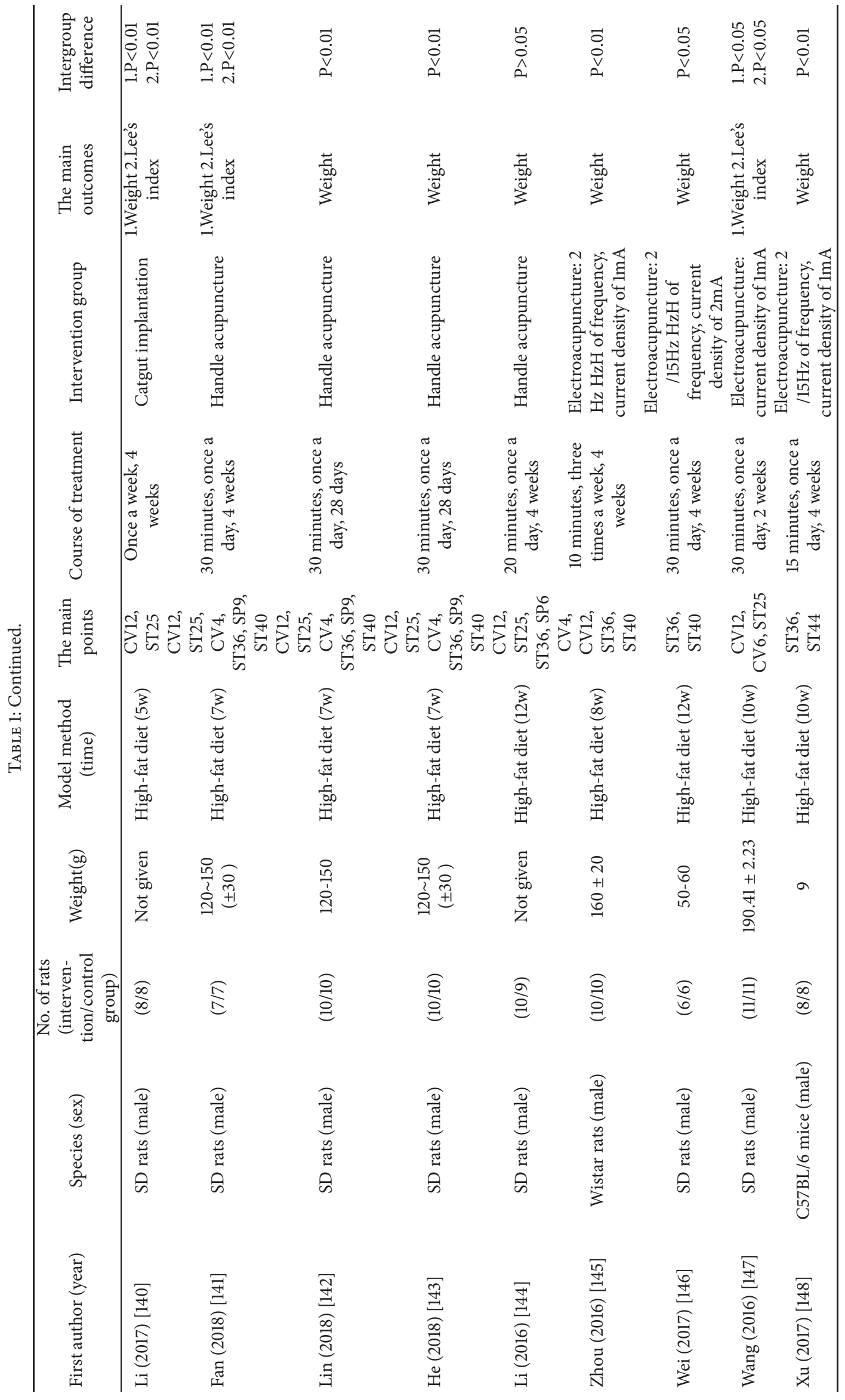


퓽

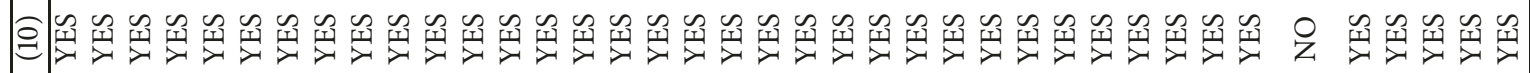

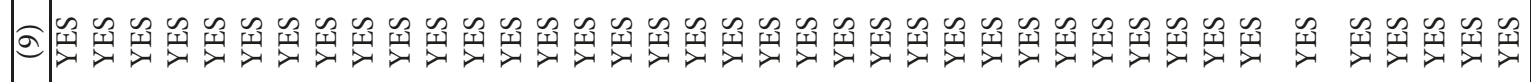

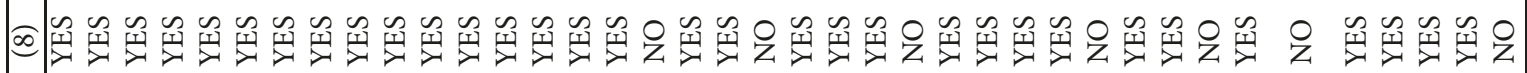

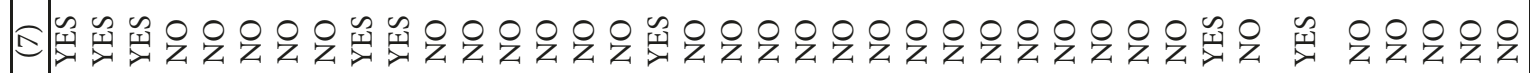

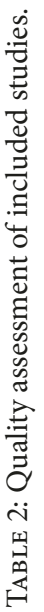

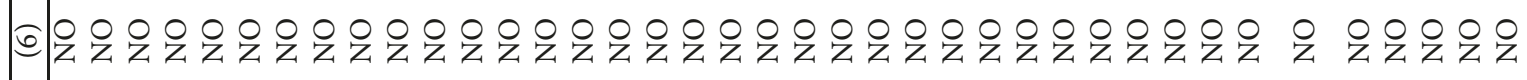

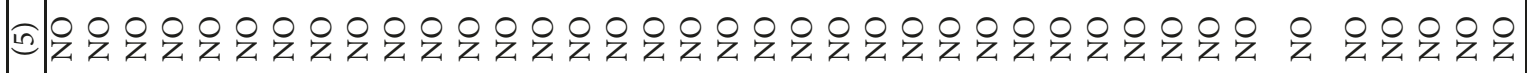

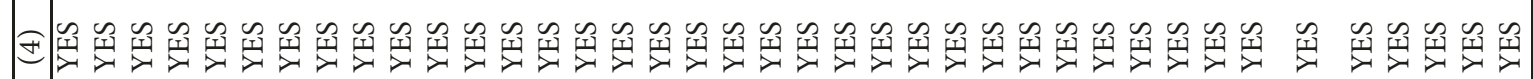

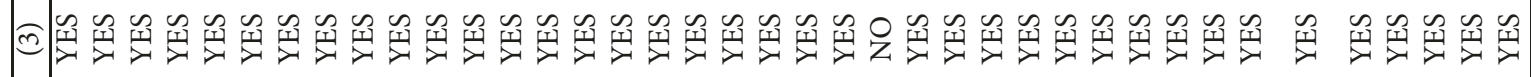

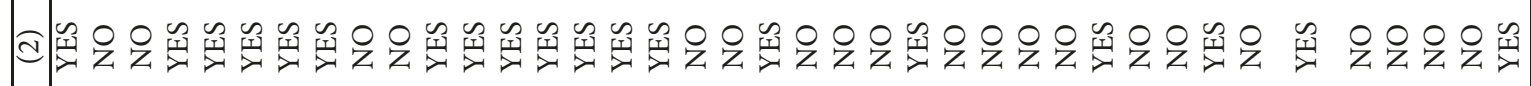

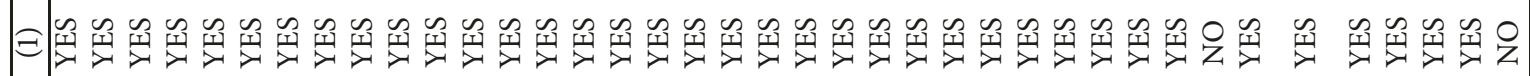

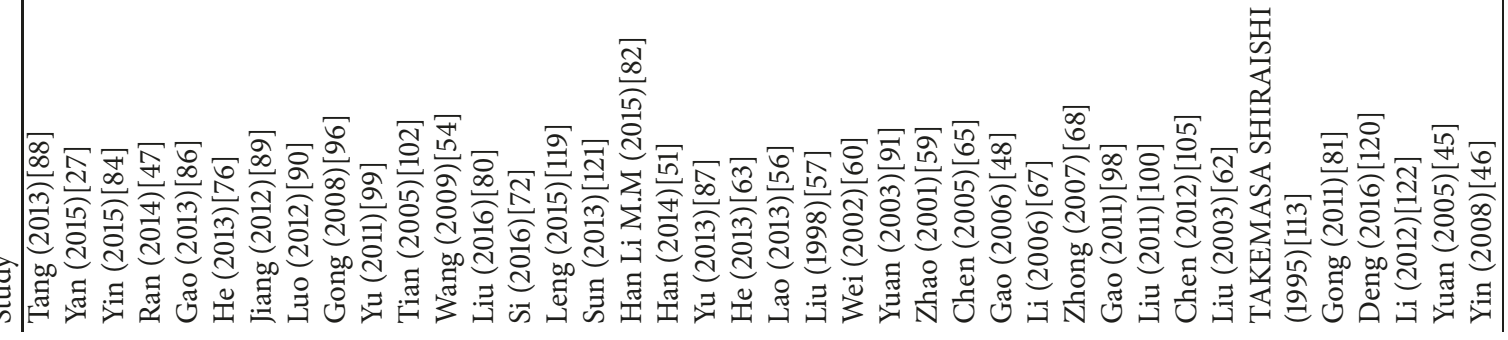




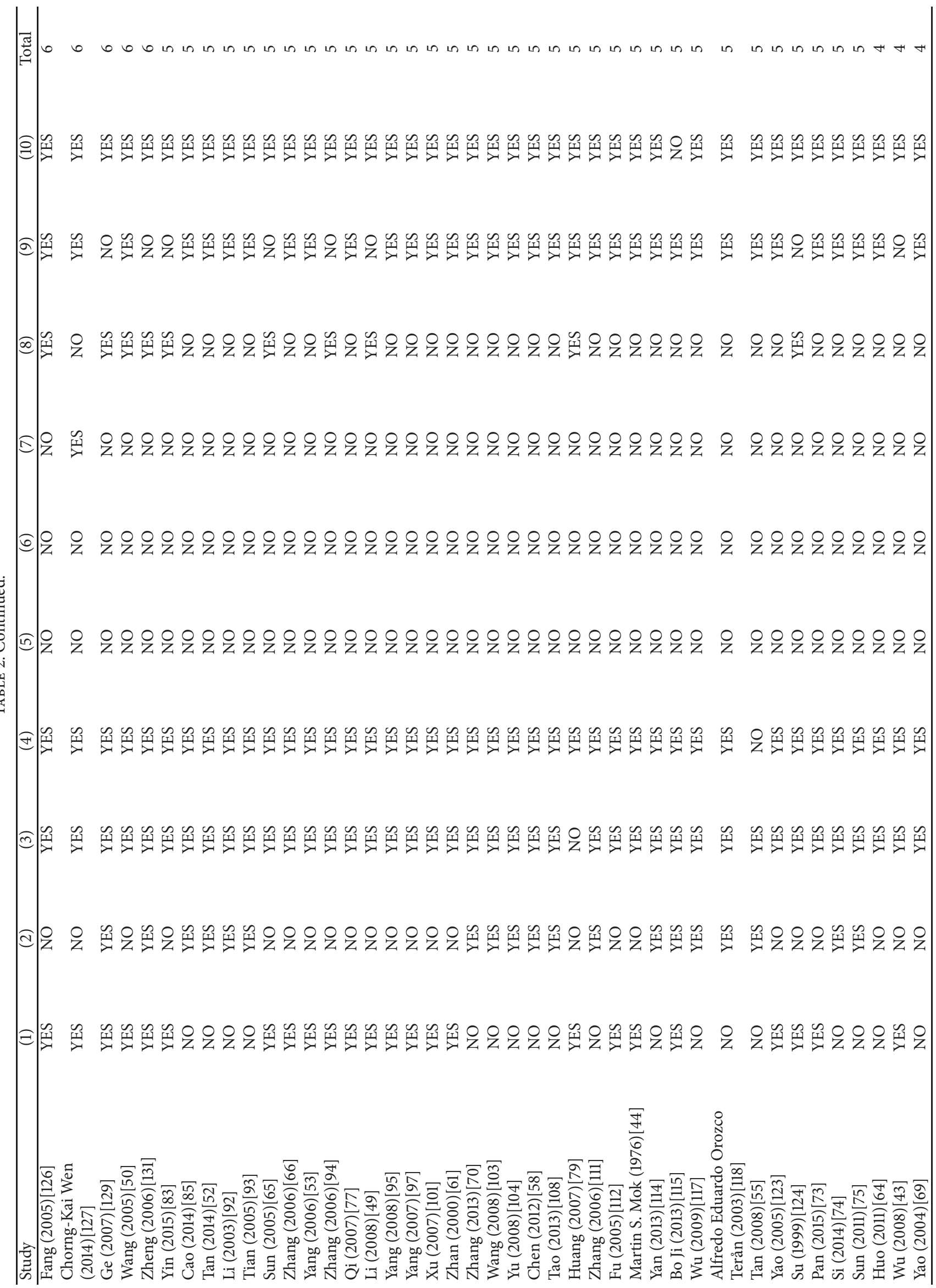




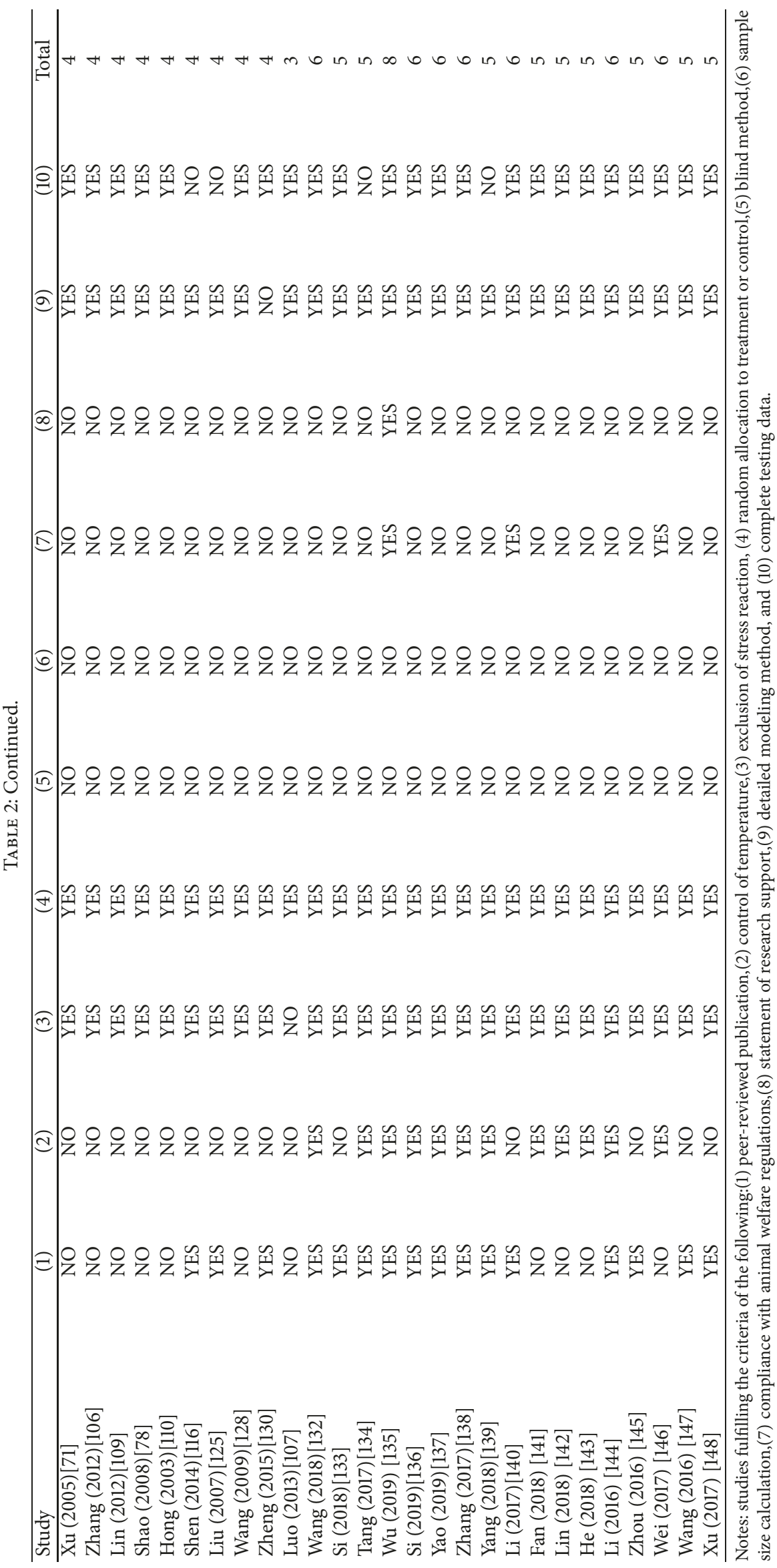




\begin{tabular}{|c|c|c|c|c|}
\hline & Acup & ouncture & & \\
\hline Study or Subgroup & Mean & SD & Total & Mean \\
\hline 1.1.1 Weight of acu & uncture gr & oup com & pare $w$ & vith contro \\
\hline Alfredo 2003 & 280.23 & 11.41 & 8 & 343.3 \\
\hline Cao 2014 & 563.53 & 37.39 & 7 & 564.37 \\
\hline Chen 2005 & 456.79 & 25.75 & 7 & 493.49 \\
\hline Chen 2012 & 452.2 & 37.19 & 10 & 588.5 \\
\hline Chen 2012\# & 512.5 & 44.15 & 8 & 573.75 \\
\hline Deng 2016 & 25.72 & 0.75 & 6 & 30.52 \\
\hline Fan 2018 & 463.85 & 11.23 & 7 & 505.14 \\
\hline Fang 2005 & 422 & 9.97 & 10 & 459.75 \\
\hline Fu 2005 & 466.8 & 14.8 & 12 & 541 \\
\hline Gao 2006 & 305.85 & 31.62 & 10 & 506.89 \\
\hline Gao 2011 & 733.94 & 23.38 & 12 & 801.84 \\
\hline Gong 2008 & 519.78 & 14.07 & 10 & 617.33 \\
\hline Gong 2011 & 572 & 7.57 & 10 & 666.9 \\
\hline Han 2014 & 343.44 & 26.97 & 10 & 388.96 \\
\hline Han Li M.M 2015 & 493.6 & 34.5 & 12 & 537.7 \\
\hline Hao 2013 & 353.2 & 12.84 & 10 & 443.3 \\
\hline He 2013 & 480.23 & 30.75 & 10 & 524.32 \\
\hline He 2013\# & 407.86 & 8.59 & 8 & 470 \\
\hline He 2018 & 380.22 & 16.479 & 10 & 482.31 \\
\hline Hong 2003 & 335.3 & 22.13 & 10 & 394.33 \\
\hline Huo 2011 & 266.83 & 19.48 & 12 & 292.64 \\
\hline Jiang 2012 & 576.6 & 9.76 & 10 & 666.9 \\
\hline Lao 2013 & 368.22 & 18.49 & 10 & 391.5 \\
\hline Leng 2015 & 416.9 & 35.7 & 7 & 489.4 \\
\hline Li 2003 & 712.5 & 1.4 & 10 & 752.7 \\
\hline Li 2006 & 424.21 & 8.16 & 20 & 506.73 \\
\hline Li 2008 & 394 & 17.8 & 8 & 471 \\
\hline Li 2012 & 340.13 & 13.35 & 15 & 366.4 \\
\hline Li 2016 & 423.67 & 13.08 & 6 & 471.17 \\
\hline Li 2017 & 380.38 & 17.05 & 8 & 414.5 \\
\hline Lin 2012 & 522.5 & 73.527 & 8 & 622.67 \\
\hline Lin 2018 & 379.5 & 17.44 & 10 & 478.5 \\
\hline Liu 1998 & 333.28 & 26.54 & 8 & 387.6 \\
\hline Liu 2003\# & 440.21 & 26.16 & 12 & 513.65 \\
\hline Liu 2016 & 514.4 & 12 & 10 & 655.4 \\
\hline Luo 2012 & 447.44 & 15.33 & 9 & 490.44 \\
\hline Luo 2013 & 501.56 & 23.41 & 11 & 404.65 \\
\hline Mok 1976 & 732 & 30 & 3 & 733 \\
\hline Pan 2015 & 308.9 & 59.02 & 10 & 361.5 \\
\hline Qi 2007 & 240.8 & 32.98 & 10 & 288.1 \\
\hline Ran 2014 & 498.58 & 34.44 & 12 & 557.68 \\
\hline Shao 2008 & 540.2 & 80.9 & 10 & 600.8 \\
\hline Si 2014 & 326.3 & 34.42 & 10 & 389 \\
\hline Si 2018 & 24.41 & 1.01 & 10 & 31.15 \\
\hline Si 2019 & 24.38 & 1.08 & 10 & 29.61 \\
\hline Su 1999 & 459.43 & 27.2 & 14 & 492 \\
\hline Sun 2005 & 440.2 & 31.27 & 12 & 513.26 \\
\hline Sun 2011 & 338.55 & 8.564 & 10 & 402.77 \\
\hline Tan 2008 & 394 & 17.8 & 8 & 471 \\
\hline Tan 2014 & 348.25 & 16.127 & 10 & 371.223 \\
\hline Tang 2013 & 28.28 & 4.3 & 6 & 36.89 \\
\hline Tao 2013 & 360.4 & 59.85 & 15 & 377.73 \\
\hline Tian 2005 & 683.5 & 13.13 & 10 & 753 \\
\hline Wang 2005 & 190.85 & 31.62 & 20 & 256.89 \\
\hline Wang 2008 & 353.5 & 27.1 & 12 & 418 \\
\hline Wang 2009 & 353.5 & 28.3 & 12 & 433 \\
\hline Wang 2009\# & 337.24 & 15.66 & 8 & 433.35 \\
\hline Wang 2016 & 479.87 & 8.53 & 11 & 524.22 \\
\hline Wang 2018 & 464.3 & 23.94 & 8 & 499.22 \\
\hline Wei 2002 & 459.43 & 27.2 & 14 & 492 \\
\hline Wei 2017 & 423.67 & 13.08 & 6 & 471.17 \\
\hline Wu 2008 & 245.98 & 12.36 & 6 & 271.83 \\
\hline Wu 2009 & 324.8 & 26.4 & 15 & 376.9 \\
\hline Wu 2019 & 417.8 & 20 & 8 & 450.8 \\
\hline Xu 2007 & 563.33 & 14.83 & 18 & 615.56 \\
\hline Xu 2017 & 24.6 & 0.37 & 8 & 30.26 \\
\hline Yan 2013 & 440.2 & 26.15 & 12 & 513.75 \\
\hline Yang 2006 & 281.12 & 29.15 & 15 & 302.4 \\
\hline Yang 2007 & 524.38 & 34.38 & 10 & 611.6 \\
\hline Yang 2008 & 510.22 & 6.46 & 8 & 560.27 \\
\hline Yao 2004 & 439.75 & 29.15 & 8 & 484.56 \\
\hline Yoo 2019 & 470.95 & 26.09 & 8 & 524.32 \\
\hline Yin 2008 & 251.76 & 12.15 & 6 & 299.2 \\
\hline Yu 2008 & 393.33 & 18.315 & 8 & 409.375 \\
\hline Yu 2013 & 506.8 & 16 & 10 & 593 \\
\hline Yuan 2003 & 422 & 9.97 & 8 & 459.75 \\
\hline Zhang 2006 & 442.1 & 30.22 & 6 & 512.24 \\
\hline Zhang 2006 * & 284.2 & 24.21 & 7 & 320.3 \\
\hline Zhang 2006\# & 326.38 & 20.26 & 8 & 393.3 \\
\hline Zhang 2012 & 334.65 & 3.68 & 8 & 434.96 \\
\hline Zhang 2013 & 287.78 & 8.059 & 8 & 328.313 \\
\hline Zhang 2017 & 37.9 & 0.67 & 10 & 42.01 \\
\hline Zhao 2001 & 411 & 18.2 & 13 & 481.8 \\
\hline Zheng 2006 & 533.375 & 58.95 & 8 & 577.143 \\
\hline Zheng 2015 & 46.7 & 2.3 & 6 & 49.34 \\
\hline Zhong 2007 & 372.2 & 20.4 & 6 & 451.8 \\
\hline & 423.67 & 13.08 & 6 & 471.17 \\
\hline Subtotal (95\% Cl) & $4 \angle 3.01$ & 13.08 & 846 & \\
\hline $\begin{array}{l}\text { Heterogeneity: } \text { Tau }^{2} \\
\text { Test for overall effec }\end{array}$ & $\begin{array}{l}1.97 ; \mathrm{Chi}^{2} \\
Z=15.05\end{array}$ & $\begin{array}{l}=507.17, \\
(P<0.000\end{array}$ & $\begin{array}{l}d f=8( \\
01)\end{array}$ & $6(P<0.00$ \\
\hline Total $(95 \% \mathrm{Cl})$ & & & 846 & \\
\hline $\begin{array}{l}\text { Heterogeneity: Tau } \\
\text { Test for overall effec }\end{array}$ & $\begin{array}{l}1.97 ; \mathrm{Chi}^{2} \\
\mathrm{Z}=15.05\end{array}$ & $\begin{array}{l}=507.17, \\
(P<0.000\end{array}$ & $\begin{array}{l}\mathrm{df}=8 \mathrm{e} \\
01)\end{array}$ & $6(P<0.00$ \\
\hline
\end{tabular}

FIGURE 2: The forest plot of outcome measure 'weight after intervention.' 


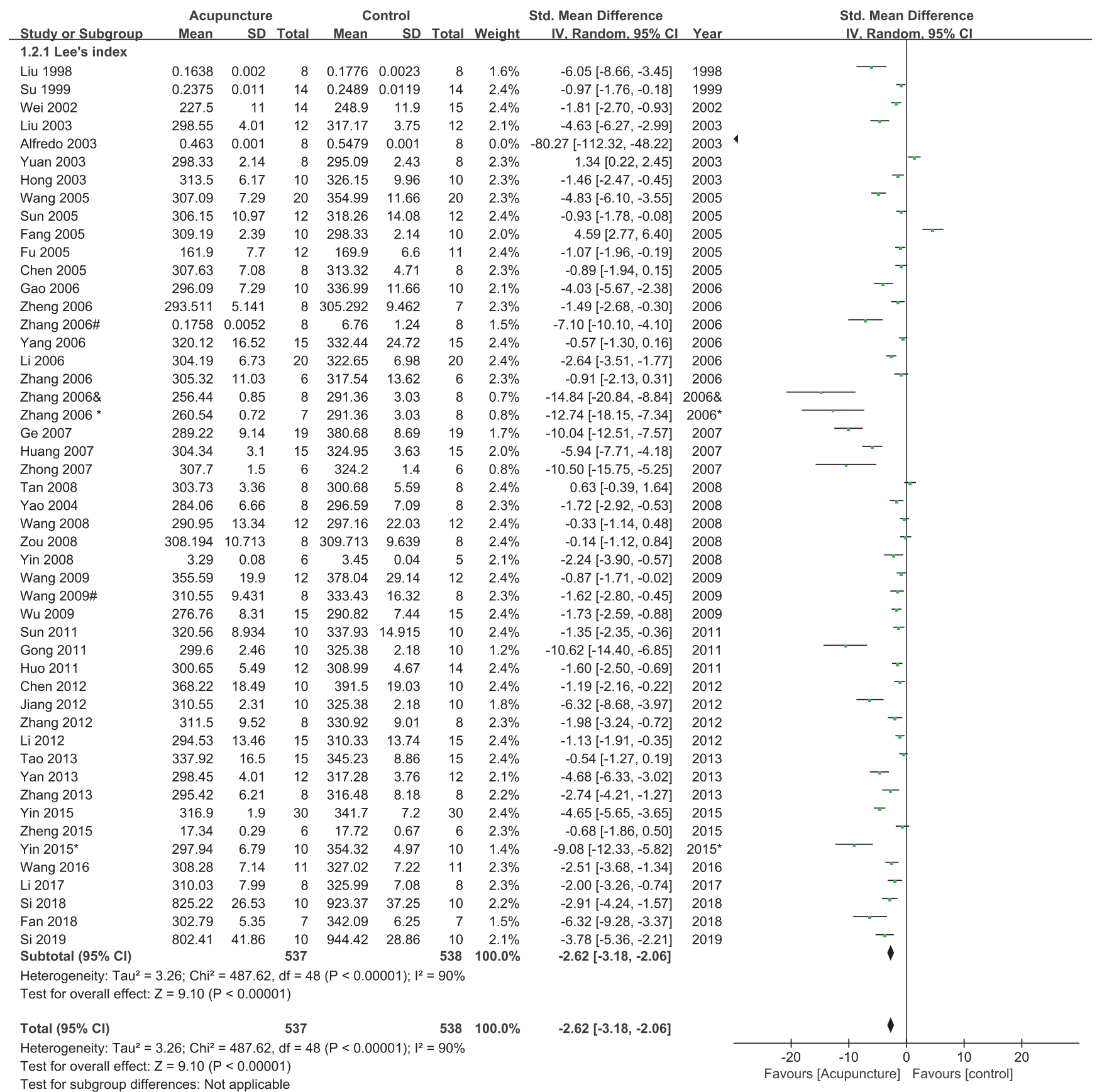

FIGURE 3: The forest plot of outcome measure 'Lee's index after intervention.'

we carried out subgroup analyses stratified by the two factors on weight of rats, respectively. A random effect was used for statistical analysis. Heterogeneity was still great in each group.

There are sixty articles compared electroacupuncture against other interventions, and the results showed high heterogeneity (heterogeneity $\mathrm{X}^{2}=354.60, \mathrm{I}^{2}=83 \%$, Figure 4 ). It also indicated that electroacupuncture was more effective than other interventions in losing weight ( $\mathrm{n}=1178$, SMD $-2.72,95 \% \mathrm{CI}$ : $-3.13 \sim-2.31 ; \mathrm{Z}=12.91, \mathrm{p}<0.00001$, Figure 4).
Handle acupuncture group versus control group was tested in 16 studies. Great heterogeneity was found in this subgroup (heterogeneity $\mathrm{X}^{2}=113.93, \mathrm{I}^{2}=88 \%$, Figure 4 ). Handle acupuncture group showed more reduction in weight compared with control group ( $\mathrm{n}=288$, SMD-2.34, 95\%CI:-3.34 $\sim-1.34 ; \mathrm{Z}=4.60, \mathrm{p}<0.00001$, Figure 4). Nine studies showed that catgut implantation tends to lose more weight than control group (heterogeneity $\mathrm{X}^{2}=35.61, \mathrm{I}^{2}=78 \%$; $=188$, SMD2.64, 95\%CI:-3.53 -1.75; $Z=5.79, p<0.00001$, Figure 4). Two studies showed that auricular acupuncture tends to lose more 


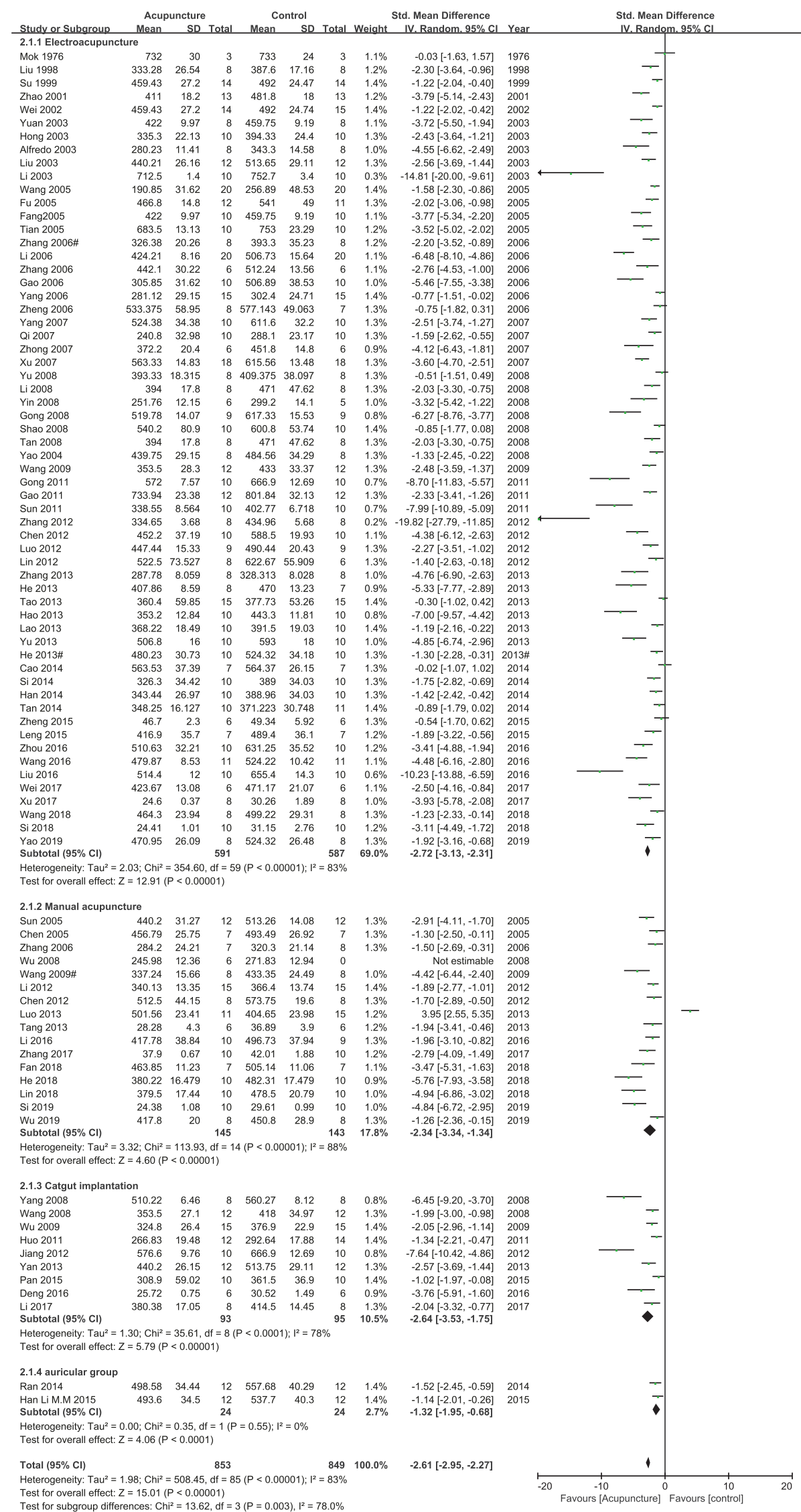

FIGURE 4: The forest plot of outcome measure 'weight after intervention' in subgroup according to different acupuncture methods. 
weight than control group (heterogeneity $\mathrm{X}^{2}=0.35, \mathrm{I}^{2}=0 \%$; $\mathrm{n}=48$, SMD-1.32, 95\%CI:-1.95 -0.687; $\mathrm{Z}=4.06, \mathrm{p}<0.0001$, Figure 4).

Based on different rat strains, the included studies were divided into four subgroups: SD rats, Wistar rats, mice, and guinea pigs. High heterogeneity was noted in both the subgroup of SD and Wistar rats but low in the mice subgroup (SD rats subgroup: $\mathrm{I}^{2}=73 \%, \mathrm{p}<0.00001$; Wistar rats subgroup: $\mathrm{I}^{2}=98 \%, \mathrm{p}<0.00001$; mice subgroup: $\mathrm{I}^{2}=37 \%$, $\mathrm{p}<0.00001$, Figure 5).

3.7. Metaregression. The meta-analysis indicated that acupuncture group was superior to control group in losing weight and controlling Lee's index; unfortunately, significant heterogeneity was observed. As a result, we adopted further multifactor metaregression to explore the sources of heterogeneity by taking acupuncture methods (handle acupuncture, electroacupuncture, and catgut implantation) and rat strains (SD rats, Wistar rats, and mice) as covariates. However, the results of metaregression showed that acupuncture methods and rat strains were irrelevant to heterogeneity (Figure 6).

3.8. Assessment of Bias. We assessed publication bias with funnel plot and Egger's and Begg's Test. The asymmetry funnel plot suggested that there was publication bias (Figure 7). Quantitative evaluation by Begg's and Egger's Test showed that significant publication exists $(\operatorname{Pr}>|z|=0.000, P>|t|=$ 0.000 , Figure 8).

\section{Discussion}

4.1. Principal Findings. To our knowledge, this is the first systematic review and meta-analysis to assess the efficacy of acupuncture for animal model of simple obesity with weight and Lee's index as the main outcome measures. This metaanalysis indicated that acupuncture could have a certain effect on simple obesity, including losing weight and reducing Lee's index, and the conclusion is similar to previous clinical metaanalysis and systematic reviews [22-26].

4.2. Possible Therapeutic Mechanism of the TCM. "Dan Brook Heart Law," one of the classical works of TCM in China, holds that obese people are more susceptible to phlegm dampness. The primary pathogenesis is internal stagnation of fluid dampness and meridian blockage by accumulated phlegm, which has close relationship with spleen, stomach, kidney, and large intestine. Therefore, eliminating dampness and phlegm through invigorating spleen is the basic principle of treatment [27]. The selected acupoints are almost all belonging to the Stomach, Spleen, and Conception Vessel $(96.25 \%)$. It is just in line with the basic pathogenesis of turbid sputum, which has a key role in ensuring effectiveness by eliminating dampness and phlegm through invigorating spleen, harmonizing Yin and Yang, and dredging channel of Qi and Blood.

Zusanli (ST 36), the most frequently used he-sea point of Stomach Meridian of Foot-Yangming, can enhance the function of spleen and stomach, eliminate phlegm, and remove dampness. Lots of experimental results illustrated that acupuncturing of Zusanli (ST 36) can promote gastrointestinal wriggle, improve gastrointestinal motility, and regulate gastrointestinal function [28, 29]. Research [30] suggested that electroacupuncture on Zusanli (ST 36) or Neiting (ST 44) could suppress appetite and stave off hunger by stimulating beta-receptors; meanwhile, acupuncture has anticholinergic functions. Neiting (ST 44), as spring point of Stomach Meridian of Foot-Yangming, is good at clearing damp heat in the stomach and intestine specially. Zhongwan (CV 12) is a front-mu point of the Stomach, as well as an influential point of fu-organs and an intersecting point of the Small Intestine, Stomach, Triple Energize, and Conception Channel. Therefore, it can be used to treat all spleen and stomach diseases by regulating activities of qi, ascending lucidity, and descending turbidity. Acupuncture in Zhongwan point is valuable to regulate gastrointestinal function and gastric acid secretion because afferent neurons in CV12 and gastrointestinal tract overlap morphologically in the spinal ganglions of T7-L2 [31]. Tianshu (ST 25), located at the abdomen, is the most common place for body fat to accumulate. So acupuncturing in Tianshu has a better local stimulation effect for promoting abdominal fat to decompose [32, 33]. As frontmu point of the Large Intestine Meridian of Hand-yangming, Tianshu (ST 25) is beneficial for clearing away the pathogenic heat of the large intestine, removing stagnancy, regulating qi, and relaxing the bowels. Therefore, Tianshu (ST 25) is a preferred acupoint for simple obesity with Stomach-intestine Excessive Heat Type, whose symptoms include constipation, lack of body fluid, and feces dry knots. As Tianshu (ST 25) is just located on the body surface projection of gut, stimulation of acupuncture more easily passes into the intestine to adjust gastrointestinal motility, absorption, and secretion. For its biphasic regulation and excellent therapeutic effects for intestinal disease, Tianshu (ST 25) has been widely used clinically [34-36]. Sanyinjiao (SP 6), an intersecting point of the Liver, Spleen, and Kidney Meridians, is an important point to treat diseases of the three relevant organs by harmonizing liver, invigorating spleen and kidney, removing dampness, and clearing away turbidness. As a result, acupuncture of Sanyinjiao can provide better curative effect for obesity caused by retention of water, dampness, phlegm, and fluid caused by liver stagnation or deficiency of spleen and kidney. Research [37] has shown that acupuncture of Sanyinjiao can reduce the content of cholesterol in the blood by restraining its synthesis and absorption, accelerating its decomposition and excretion, and changing its distribution in the plasma and tissue. Guanyuan ( $\mathrm{CV} 4$ ), located on 3 inches below the navel and right at the pubic region where the root of human life is originated, is very effective in curing any injury detriment of Primordial Qi. Through reinforcing vitality and strengthening primordial qi, promoting blood circulation to resolve turbid-phlegm, Guanyuan is especially suitable for simple obesity resulting from the deficiency of Qi. Its curative effect is confirmed by many researches $[38,39]$.

4.3. Possible Mechanism from Modern Medicine Aspect. Combining the above analysis and numerous research results [12, 


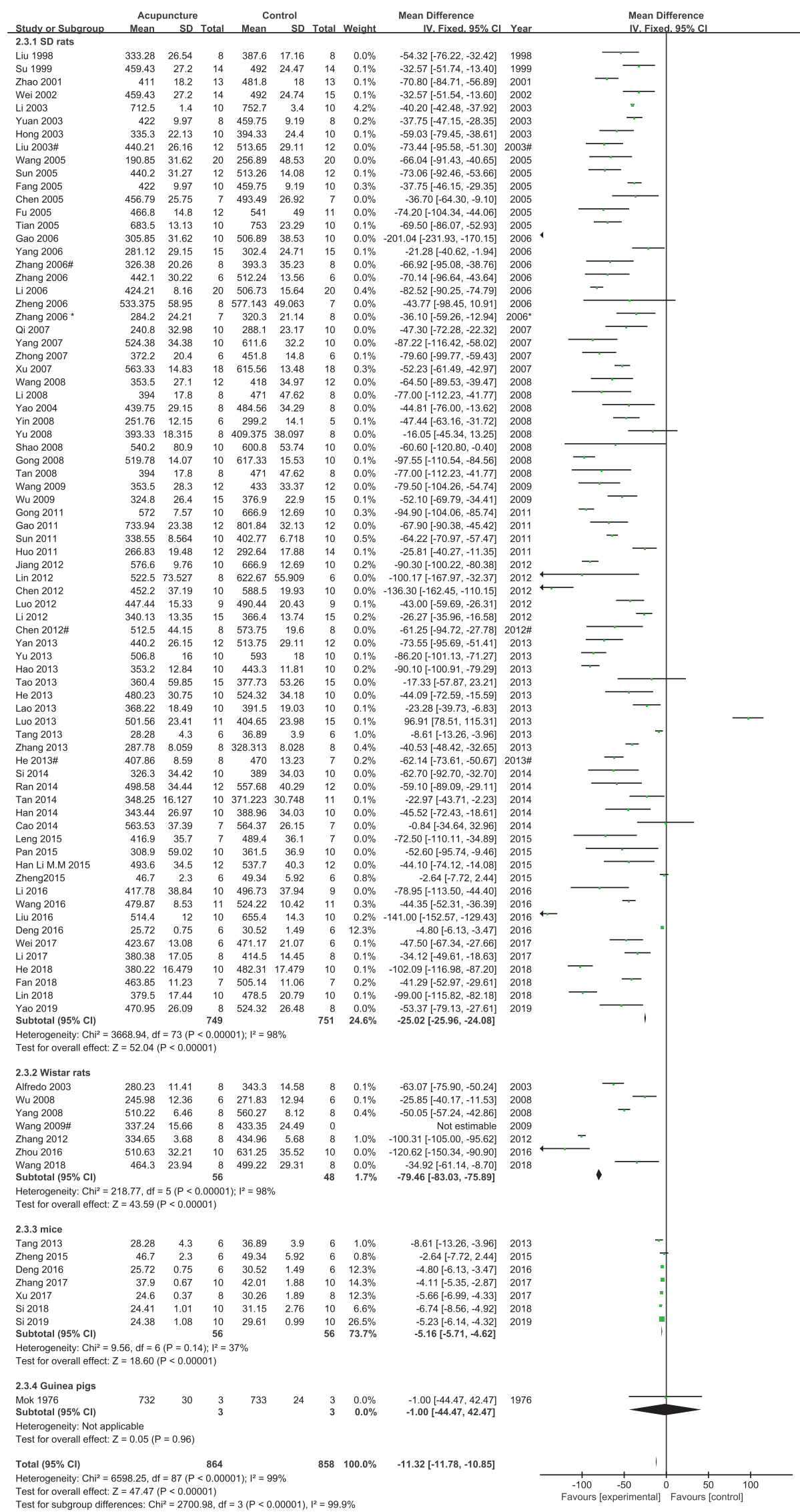

FIGURE 5: The forest plot of outcome measure 'weight after intervention' in subgroup according to different species. 


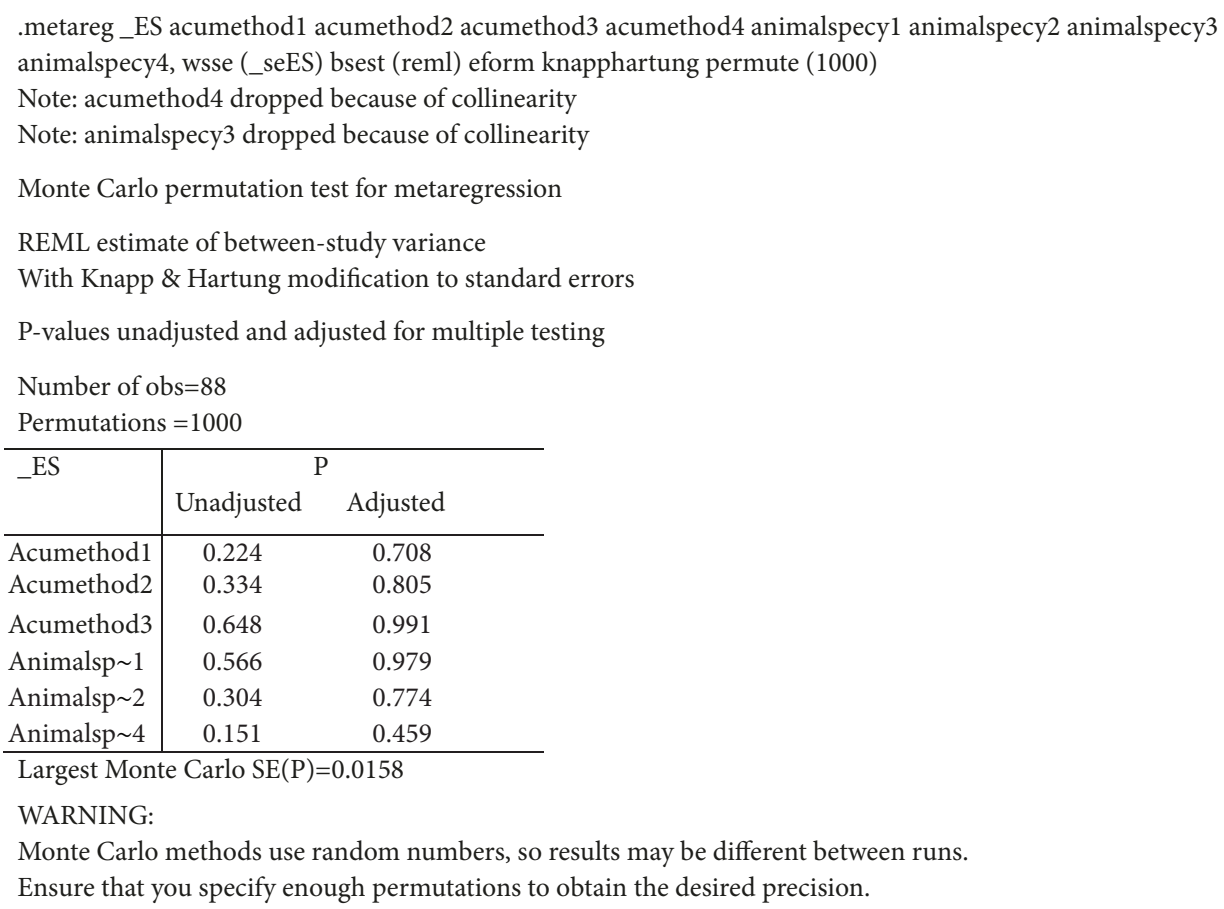

FIgURE 6: The results of the meta-regression employed acupuncture methods and rat strains as covariates.

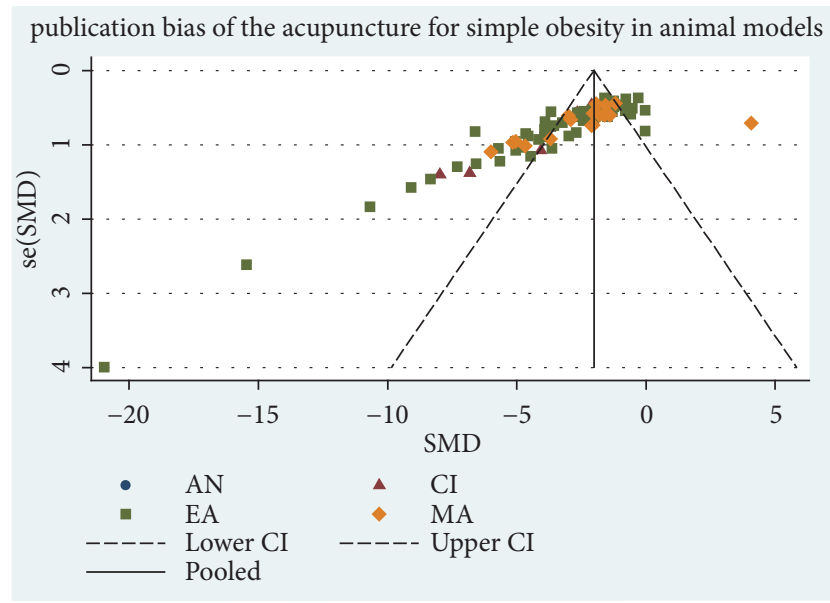

FIGURE 7: Funnel plot for publication bias analysis.

40, 41], the possible modern mechanism of acupuncture for simple obesity includes but is not limited to the following. Acupuncture can inhibit excitatory digestion in stomach and intestine and suppress appetite in obese patients. On the other hand, acupuncture can promote energy metabolism and increase energy expenditure to accelerate lipolysis. The included animal experiments in this paper also revealed the role of acupuncture in treating simple obesity. Acupuncture has optimal regulation on body's weight [42-46], lipid metabolism [47-50], blood fats [51-55], insulin [56-58], electrogastrogram (EGG) [27], and nucleus of hypothalamus. The possible central mechanisms of acupuncture for simple obesity are as follows: (1) neurons discharge regulation: acupuncture can reduce the excitation of lateral hypothalamic area (LHA) and increase electric activity frequency in ventromedial hypothalamus (VNH). So acupuncture could achieve weight loss through appetite suppressing and calories cutting [57, 59]; (2) modulation of the neurotransmitter: acupuncture can normalize hypothalamus ingestion central functions by adjusting content of monoamine neurotransmitters including catecholamines and 5-hydroxytryptamine (5-HT) [60, 61], cholecystokinin (CCK) and vasoactive intestinal peptide (VIP) [62], neuropeptide Y (NPY), and leptin (LP) [27, 54, 62-75]; (3) improving insulin resistance: acupuncture can increase the number and affinity of insulin receptor to improve insulin resistance (IR) status. So it is advantageous for reversing the metabolic disorders of glucose and lipids $[56,58,76-82]$. However, the conclusion of this meta-analysis still should be taken prudently due to the publication bias, significant heterogeneity, and other limitations.

4.4. The Value of Animal Studies and Systematic Review of the Effectiveness of Acupuncture for Obesity in Animal Model. It is well known that acupuncture is safe and effective for obese people to some extent. But controversy does exist for acupuncture's placebo effect. Revealing the mechanisms of acupuncture for obesity and making sure that the effect is not just placebo are significant for the development of acupuncture. To uncover these puzzles, it is necessary to objectively study the effects of acupuncture on fat and related tissues, cells, and metabolic signal pathways. As we know, it is unethical and impossible to get human's tissues and cells optionally. So, animal studies of acupuncture for obesity are of great value and cannot be replaced completely by clinical research. It should be noticed that all animal studies 


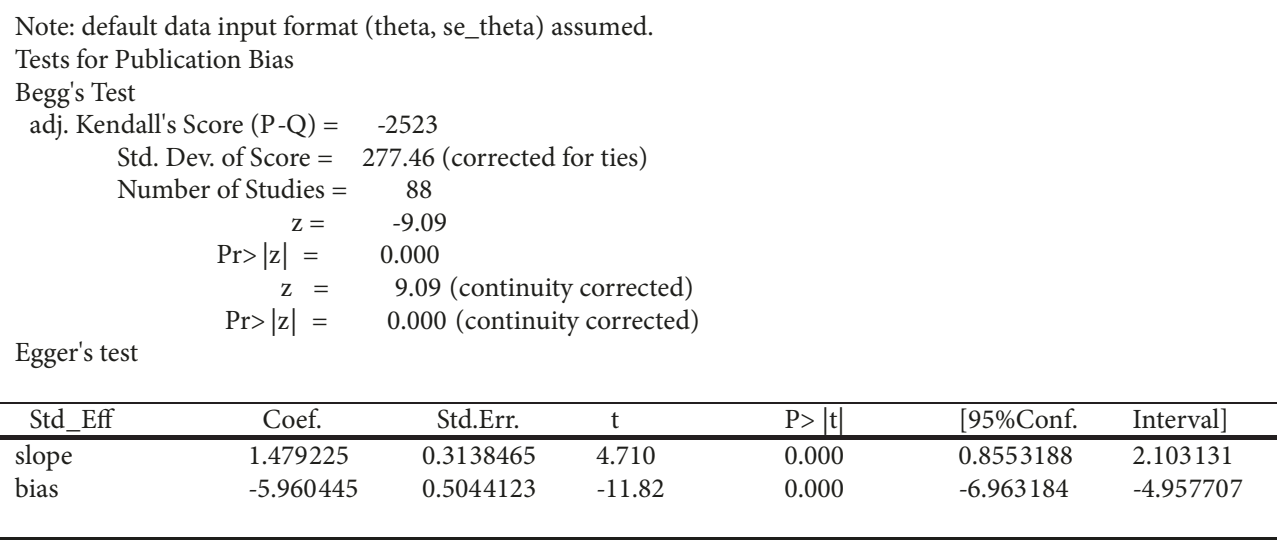

FIGURE 8: Egger's and Begg's Test for publication bias analysis.

for potential mechanism of obesity treated by acupuncture depend on a precondition: acupuncture is effective for obese animals definitely. In general, applying the method of systematic evaluation to evaluate the experimental research of acupuncture is of great significance for improving the basic research level and quality of acupuncture. Therefore, we conduct this systematic review and meta-analysis to assess the effectiveness of acupuncture in treatment of simple obesity in animal models.

4.5. Limitations. Firstly, the vast majority of the included studies were published in Chinese; few studies were published in English. We did not include studies in other languages, which may cause certain degree of publication bias. Most of the included studies came from China because acupuncture as a key component of TCM is widely used in China but not so popular in other countries.

Secondly, no 'negative' trial was included in our metaanalysis, which may induce potential publication bias. This might be because negative data is hard to publish compared with positive data. As a result, we may miss some negative data, which could lead to an overestimate of the effectiveness of acupuncture for simple obesity.

Thirdly, the methodological weakness of included trials also prevented drawing definitive conclusions as follows. (1) Randomization: in all the trials, we selected and adopted the principle of randomization, but the details of randomization procedures were often absent. That is why we could not give direct judgement. (2) Blinding: only one of the enrolled trials used blinding method, which may cause bias to some extent. (3) Low quality and small numbers of studies: low quality of the included articles and small number of studies with specific acupuncture methods (such as auricular acupuncture) were certain limitations to our analysis. (4) Lacking unified diagnostic criteria: to our knowledge, acupuncture is a key component of TCM, so it is best to apply acupuncture based on TCM syndrome. However, a considerable part of the included studies used "modern diagnosis" instead of "TCM syndrome" as the diagnostic criteria.

Besides, the substantial heterogeneity should be taken into consideration. Despite sensitivity analysis, subgroup analysis, and multifactor metaregression all performed, the factors that caused high heterogeneity have not been found yet. There are many reasons contributing to the generation of heterogeneity, such as the intramuscular needle insertion with different angles, depths and locations, different types of needles, different needling techniques of different operators, time of retaining needle, and scheduled time interval of acupuncture.

4.6. Implications for Future Trials. Further studies should recognize and overcome these referred limitations by the following aspects. Firstly, future studies should be encouraged to post negative data, side effects, and complications to reflect the effect of acupuncture objectively.

Also, rigorously designed RCTs and improved methodology are required. Randomization and blinding should be performed strictly. Future trials should attempt to expand sample size of trials with special acupuncture and adopt more unified TCM diagnostic criteria.

Moreover, a unified standard of acupuncture therapy should be taken seriously since details of acupuncture manipulation closely are related to the efficacy. Standardized acupuncture protocols, acupuncture techniques, treatment course, outcome measures, and even highly skilled acupuncturist are necessary.

\section{Conclusion}

Although our review provided positive evidence that acupuncture seems to be an effective therapy for simple obesity, it should be noted that the evidence justifies more future high-quality studies due to the possibility of publication bias and significant heterogeneity of the included studies.
Abbreviations
RCTs: Randomized controlled trials
CNKI: China National Knowledge Infrastructure
VIP: Chinese Science and Technology Periodical Database 
CBM: Chinese Biology Medicine Disc

RR: Relative risk

SMD: Standard mean difference

CI: Confidence interval

BMI: Body mass index

SD: Sprague Dawley

TCM: Traditional Chinese Medicine

NPY: Neuropeptide Y

LP: Leptin

EGG: Electrogastrogram

LHA: Lateral hypothalamic area

VNH: Ventromedial hypothalamus

5-HT: 5-Hydroxytryptamine

CCK: Cholecystokinin

VIP: Vasoactive intestinal peptide

IR: Insulin resistance.

\section{Data Availability}

The datasets supporting the conclusions of this article are included within the article.

\section{Disclosure}

The funders do not have any role in study design, data collection and analysis, decision to publish, or preparation of the manuscript.

\section{Conflicts of Interest}

The authors declare that they have no conflicts of interests.

\section{Authors' Contributions}

Xiao-lin Fan and Sheng-feng Lu conceived and designed the experiments. Xiao-lin Fan and Mei-ling Yu performed the experiments. Mei-ling Yu, Shu-Ping Fu, and Sheng-feng $\mathrm{Lu}$ analyzed the data. Xiao-lin Fan wrote the paper. Shengfeng Lu, Mei-ling Yu, Yi Zhuang, and Shu-Ping Fu revised the manuscript. Xiao-lin Fan and Mei-ling Yu contributed equally to this work. All authors have read and approved the final manuscript.

\section{Acknowledgments}

This work was supported by Grants from the National Natural Science Foundation of China (Nos. 81303019 and 81574062), Major Projects of Natural Science Research in Colleges and Universities of Jiangsu Province (16KJA360003), Six Talent Peaks Project in Jiangsu Province (YY-033), Qinglan Project of Jiangsu Province (2016), and 333 High Level Talents Training Project of Jiangsu Province.

\section{References}

[1] The GBD 2015 Obesity Collaborators, "Health effects of overweight and obesity in 195 countries over 25 years," The New England Journal of Medicine, vol. 377, no. 1, pp. 13-27, 2017.
[2] Z. Y. Lu and N. S. Zhong, Internal Medicine, vol. 807, People's Publishing House, Peking, China, 2008.

[3] Q. B. Ouyang, X. L. Liao, J. Sun, and L. F. Zhang, "Observation on effects of acupuncture clinical treatment to 67 cases of obesity patients under accumulation of heat in the intestines and stomach," Progress in Modern Biomedicine, vol. 12, no. 23, pp. 4447-4450, 2012.

[4] J. Q. Zhang, L. Xu, and Y. Q. Mi, "Guide to the treatment of obesity proposed by the American endocrine society in 2015," Global Journal of Endocrinology and Metabolism Studies, vol. 36, no. 1, pp. 68-70, 2016.

[5] C. Lu-ying, "Advances in research of the pharma-therapy for obesity," Chinese Pharmaceutical Journal, vol. 37, no. 11, pp. 812814, 2002.

[6] D. P. Li, L. H. Kong, and R. Qin, "Mate-analysis of curative effect of simple obesity by acupuncture," Journal of Hubei University of Chinese Medicine, vol. 16, no. 4, pp. 100-102, 2014.

[7] L. Sai, H. Z. Zhang, and S. Y. Su, "Review and prospects of drug therapy for obesity," Clinical Journal of Diabetes World, vol. 4, no. 7, pp. 318-327, 2010.

[8] Z. Y. Chen, H. X. Zhang, and T. F. Zhang, "The acupuncture therapy and the mechanistic devlopment of simple obesity," Journal of Clinical Acupuncture \& Moxibustion, vol. 24, no. 5, pp. 42-44, 2008.

[9] X. Zhang, Acupuncture: Review and Analysis of Reports on Controlled Clinical Trials, World Health Organisation, 2002.

[10] Y. Shi, C. Zhao, and X.-Y. Zuo, "Clinical study on treatment of simple obesity due to spleen deficiency by acupuncturemoxibustion," Journal of Acupuncture and Tuina Science, vol. 6, no. 6, pp. 352-355, 2008.

[11] Y.-H. Zhang, "Treatment of 35 cases with type 2 diabetes mellitus by acupuncture-moxibustion therapy," Journal of Acupuncture and Tuina Science, vol. 8, no. 5, pp. 300-301, 2010.

[12] G.-Y. Wang and B.-W. Al, "Thinking about increasing therapeutic effect of acupuncture and moxibustion for slimming," Zhongguo Zhen Jiu = Chinese Acupuncture \& Moxibustion, vol. 26, no. 7, pp. 527-529, 2006.

[13] Z. C. Liu, F. M. Sun et al., "Effect of acupuncture on level of monoamins and activity of adenosine triphosphatase in lateral hypothalamic area of obese Rats," Chinese Journal of Integrated Traditional and Western Medicine, vol. 20, no. 7, pp. 521-523, 2000.

[14] Z. C. Liu, F. M. Sun, J. Su et al., "The research of acupuncture on the central effect of the hypothalamus of experimental obese rats," Journal of Traditional Chinese Medicine, vol. 41, no. 1, pp. 25-26, 2000.

[15] Z. C. Liu, F. M. Sun, J. Su et al., "Influence of acupuncture on leptin and insulin resistance in simple obesity," Chinese Journal of Clinical Rehabilitation, vol. 8, no. 3, pp. 562-565, 2004.

[16] M. W. Wang, Y. J. Wang et al., "Clinical observation on treatment of simple obesity by acupuncture and moxibustion in 80 cases," Journal of Traditional Chinese Medicine and Chinese Material Medica of Jilin, vol. 23, no. 6, 38 pages, 2003.

[17] Y. Y. Ren, "Abdomen electrical needle in the treatment of simple obesity: curative effect observation of 136 cases," Modern Traditional Chinese Medicine, vol. 27, no. 2, pp. 46-47, 2007.

[18] S. H. Cui, G. Y. Li et al., "Observation on anricular-point plaster on children with simple obesity," International Journal of Triditional Chinese Medicine, vol. 32, no. 1, pp. 66-67, 2010.

[19] J. Li and F. Yang, "Cupping therapy in the treatment of simple obesity. Curative effect observation of 37 cases," Shanxi Journal of Traditional Chinese Medicine, vol. 30, no. 6, pp. 716-717, 2009. 
[20] H. Y. Zhao, B. W. Ai et al., "Clinical observation on treating simple obesity by acupoint catgut embedding," Clinical Journal of Chinese Medicine, vol. 7, no. 4, pp. 41-43, 2015.

[21] P. Pound and I. Roberts, "The need for systematic reviews of animal studies," Chinese Journal of Evidence-Based Medicine, vol. 5, no. 1, pp. 3-5, 2005.

[22] B. Li, Y. H. Du, and J. Xiong, "Systematic evaluation of therapeutic effect of acupuncture for treatment of simple obesity," Chinese Acupuncture Moxibustion, vol. 29, no. 10, pp. 856-860, 2009.

[23] Z. Yu, C. H. Ju, B. Xu et al., "Meta-analysis on acupuncture for treatment of simple obesity," Lishizhen Medicine and Materia Medica Research, vol. 21, no. 2, pp. 434-436, 2010.

[24] D. P. Li, L. H. Kong, R. Tan et al., "Mate- analysis of curative effect of simple obesity by acupuncture," Journal of Hubei University of Chinese Medicine, vol. 16, no. 4, pp. 100-102, 2014.

[25] Y. Y. Xia, Acupuncture for Obesity: A Systematic Review [Master's, thesis], Chengdu University of Chinese Medicine, 2015.

[26] J. Kim, K. V. Trinh, J. Krawczyk, and E. Ho, "Acupuncture for obesity: a systematic review," Journal of Acupuncture and Tuina Science, vol. 14, no. 4, pp. 257-273, 2016.

[27] Z.-K. Yan, Z.-J. Yang, and F. Chen, "Effect of electroacupuncture stimulation of "Housanli" (ST 36) and "Zhongwan" (CV 12) on serum leptin and hepatocellular JAK 2-STAT 3 signaling in obese rats," Acupuncture Research, vol. 40, no. 1, pp. 1-5, 2015.

[28] G. L. Liu, X. D. Zhang, and X. Z. Zheng, "The study of the relationship between the regulation of electroaucpumcture on electrogastrogastrography of rabbits and ARC," Jounal of Mudanjiang Medical College, vol. 22, no. 1, pp. 5-7, 2001.

[29] D. D. Luo, X. J. Yang, and S. C. An, "The study of the relationship between the regulation of electroacupuncturing Znsanli point on gastric motility and nucleus raphes magnus," Journal of Shaanxi Normal University (Natural Science Edition), vol. 37, no. 1, pp. 60-64, 2009.

[30] C. Ma and Z. C. Liu, "The study of electroacupuncturing with an inhibitive effect on stimulated of lateral hypothalamic (LHA) induced hyperactivity of rabbit's stomach," Acupuncture Research, vol. 19, no. 2, pp. 42-46, 1994.

[31] W. X. Zhan, C. B. Liu, H. L et al., "Clinical application and study of zhongwan (CV 12) acupoint in the past and present," Acupuncture Research, vol. 31, no. 5, pp. 311-313, 2006.

[32] L. M. Wu, "Research overview weight-losing by acupuncture acupoint application," Journal of Clinical Acupuncture and Moxibustion, vol. 25, no. 9, pp. 55-57, 2009.

[33] L. J. Meng, X. P. Meng, X. L. Gao et al., "Electroacupuncturing for 180 cases of female simple obesity," Chinese Journal of Tissue Engineering Research, vol. 6, no. 23, p. 3536, 2002.

[34] B. Zhu, "Biological significance of bidirectional regulation of acupuncture," World Chinese Medicine, vol. 8, no. 3, pp. 241-244, 2013.

[35] C. Zhang, Z. Shi, Q. F. Huang et al., "Commentary on indications for and the efficacy of point tianshu on basis of traditional chinese medical acupuncture-moxibustion information databank," Shanghai Journal of Acupuncture and Moxibustion, vol. 33, no. 5, pp. 384-387, 2014.

[36] L. S. Zhang and Y. Shi, "Tianshu point applied in treating bowel disease with acupuncture," Jiangxi Journal of Traditional Chinese Medicine, vol. 35, no. 12, pp. 53-56, 2004.

[37] T. F. Chen, "Treating simple hyperlipemia with acupuncture at sanyinjiao (SP 6)," Heilongjiang Journal of Traditional Chinese Medicine, no. 1, pp. 43-44, 2003.
[38] L. L. Huang, Q. W. Yang, and S. X. Wang, "Bo's abdominal acupuncture in the treatment of simple obesity," Journal of Clinical Acupuncture and Moxibustion, vol. 27, no. 5, pp. 14-17, 2011.

[39] J. Tong, H. Yao, and J. X. Chen, "Clinical observation on the therapeutic effect of abdominal acupuncture therapy for simple obesity," Acupuncture Research, vol. 31, no. 3, pp. 176-178, 2006.

[40] Q. L. Wei and Z. C. Liu, "Progresses in the study on mechanisms of acu-moxi treatment of simple obesity," Acupuncture Research, vol. 25, no. 4, pp. 309-312, 2000.

[41] F. M. Sun, Z. C. Liu, M. H. Zhu, and Y. Z. Wang, "Effect of acupuncture on energy metabolism in simple obesity," International Journal of Clinical Acupuncture, vol. 8, pp. 123-128, 1997.

[42] J. H. Chen, J. M. Cui, Y. Wang et al., "Effect of acupuncture and adjustment of dietary structure against obesity: Is single action or synergistic action better?" Chinese Journal of Clinical Rehabilitation, vol. 9, no. 23, pp. 31-33, 2005.

[43] J. Z. Wu, "The effects of different acupuncture methods on the weight of obese rats," Chinese Journal of Traditional Medical Science and Technology, vol. 15, no. 5, p. 383, 2008 (Chinese).

[44] M. S. Mok, L. N. Parker, S. Voina, and G. A. Bray, "Treatment of obesity by acupuncture," American Journal of Clinical Nutrition, vol. 29, no. 8, pp. 832-835, 1976.

[45] J. H. Yuan, B. Xu, Z. C. Liu et al., "Character of a change in body weight in acupuncture reduction of weight," Shanghai Journal of Acupuncture \& Moxibustion, vol. 24, no. 5, pp. 3-5, 2005.

[46] X. Z. Yan, Acupuncture Treatment of Obesity Literature Research and Clinical Observation of Diet-Induced Obesity Rats [Master's, thesis], Shanghai University of Chinese Medicine, 2008.

[47] H. X. Ran, Q. Q. Tang, and Y. P. Wang, "Effect of electroacupuncture(EA) stimulation of auricular concha region(ACR) on dietary-induce obesity in Rats," Chinese Journal of Basic Medicine in Traditional Chinese Medicine, vol. 20, no. 3, pp. 372374, 2014.

[48] J. Z. Gao, H. F. Hou, and Y. Li, "Effect of acupuncture on lipid metabolism in Simple obese rat," Journal of Xinxiang Medical College, vol. 23, no. 5, pp. 444-445, 2006.

[49] H. Y. Li, Y. Q. Tan, and G. Z. Liang, "Effects of electroacupuncture at Zusanli and Sanyinjiao Acupoints on fat metabolism index in obese rats," in Proceedings of the Annual Conference of the Beauty of Traditional Chinese Medicine Branch of China Association of Chinese Medicine in 2008, 2008.

[50] S. J. Wang, X. L. Tian, and S. B. Kang, "Effects of acupuncture on lipometabolism of obese rats induced by glutamicsodium and its relevant factors," Hn Jornal of Gronology, vol. 25, no. 3, pp. 306-308, 2005.

[51] Z. M. Han, Y. D. Hou, L. P. Yan et al., "Effects of electroacupuncture at abdominal points such as Tianshu on the blood lipids and serum adiponectin of rats with simple obesity," Journal of Shanxi College of Traditional Chinese Medicine, vol. 15, no. 4, pp. 15-17, 2014.

[52] J. F. Tan, The Research on the Influence of Electroacupuncture Effect of Simple Obesity Rat Blood Lipid Metrobolism [Master's thesis], Chengdu University of Chinese Medicine, 2014.

[53] C. Z. Yang, S. X. Sun, C. F. Liu et al., "The effect of acupuncture on the weight and blood lipid of obese rats," Journal of clinical acupuncture and moxibustion, vol. 22, no. 12, pp. 58-59, 2006.

[54] S. H. Wang and Y. H. Li, "Effects of catgut-embedding at acupoint on contents of leptin and blood fat in obese rats," Tianjin Journal of Traditional Chinese Medicine, vol. 26, no. 1, pp. 63-65, 2009. 
[55] Y. J. Tan, Influence of Electro-acupuncture at Sanyinjiao Point on Lee's Index and Lipid Metabolism in Rates with Simple Obesity [Master's, thesis], Guangxi University of Chinese Medicine, 2008.

[56] J. X. Lao and C. Deng, "The intervention of acupuncture on insulin resistance in obese rats," Chinese Journal of Gerontology, vol. 33, no. 5, pp. 1090-1091, 2013.

[57] Z. Liu, "The experimental study on acupuncture treatment of simple obesity in rats," Acupuncture Research, vol. 1, no. 24, pp. 68-75, 1998.

[58] J. L. Chen, The Effect of Acupuncture on Insulin-Resistance in Obese Rates [Master's, thesis], Guangzhou University of Chinese Medicine, 2008.

[59] M. Zhao, J. H. Yuan, and J. Li, "Effect of acupuncture on feeding center of hypothalamus in experimental fat rats," Chinese Acupuncture \& Moxibustion, vol. 21, no. 5, pp. 305-307, 2001.

[60] Q. L. Wei and Z. C. Liu, "Effects of acupuncture on monoamine neurotransmitters in raphe nuclei in obese rats," JTCM, vol. 23, no. 2, pp. 147-150, 2003.

[61] X. Q. Zhan and Y. Han, "Weight-reducing action of acupuncture and approach to its mechanisms in rats of obesity," Chinese Acupuncture \& Moxbustion, vol. 20, no. 7, pp. 437-438, 2000.

[62] Z. C. Liu, F. M. Sun, D. H. Zhao et al., "Influence of acupuncture on leptin and insulin resistance in simple obesity," Chinese Journal of Clinical Rehabilitation, vol. 21, no. 1, pp. 40-43, 2003.

[63] R. S. He, C. H. Fang, C. Chang et al., "The effect of acupuncture on the expression of leptin and neuropeptide $\mathrm{Y}$ in obesity Rats," Journal of Emergency in Traditional Chinese Medicine, vol. 22, no. 2, pp. 255-256, 2013.

[64] X. H. Huo, Affection of blood grease, the level of serological leptin and fat accumulate by acupoint catgut [Master's thesis], Xinjiang Medical University, 2008.

[65] Z. Sun, Z. C. Zhang, and L. Ma, "Effects of acupuncture on appetite regulating factors NPY and leptin of obese rats," Chinese Journal of Behavioral Medical Science, vol. 14, no. 12, pp. 1057-1059, 2005.

[66] Y. S. Zhang, G. P. Luo, and X. S. Lai, "Affection of serological leptin by crash diet and acupuncture treatment on experimental simple obesity rats," Journal of Clinical Research, vol. 23, no. 6, pp. 837-839, 2006.

[67] X. L. Li, D. Xi, R. Y. Liu et al., "Effect of acupuncture on fat accumulation and leptin expression in the fat rats," Journal of JiangXi University of Traditional Chinese Medicine, vol. 18, no. 4, pp. 34-35, 2006.

[68] G. W. Zhong, W. Li, Z. Y. Li et al., "Effects of electroacupuncture on the expressions of leptin and neuropeptide Y protein in the hypothalamus of rats with simple obesity," Journal of Clinical Rehabilitative Tissue Engineering Research, vol. 11, no. 21, pp. 4151-4154, 2007.

[69] Q. L. Yao, A. Parhat, and G. Z. Yin, "Effect of acupuncture on leptin-hypothalamic NPY chain in obese rat," Journal of Xinjiang Medical University, vol. 31, no. 1, pp. 28-30, 2008.

[70] S. Zhang, Electroacupuncture different acupuncture point groups on the model of simple obesity rats blood lipids, insulin and leptin levels [Master's, thesis], Liaoning University of Chinese Medicine, 2013.

[71] R. X. Xu, The Influence of Acupuncture on Serum Leptin and the Relevant Indexes in Rats with Diet-Induced Obesity [Master's, thesis], Guangzhou University of Chinese Medicine, 2005.

[72] Y. C. Si, W. N. Miao, and X. F. Wang, "The effect of acupuncture with strengthening spleen and nourishing Qi on the Leptin, adiponectin, and fat cells in obese rats," Lishizhen Medicine and Materia Medica Research, vol. 27, no. 8, pp. 2016-2018, 2016.

[73] H. H. Pan and J. B. Zhao, "Influence of acupoint catgut embedding on leptin and fat accumulate in simple obesity rats," Clinical Journal of Chinese Medicine, vol. 7, no. 14, pp. 1-3, 2015.

[74] Y. C. Si, Effect of Electronic Acupuncture Abdominal Point on Abdominal Fat Mass, Leptin and Free Fatty Acids in Obesity Rates [Master's, thesis], Shanxi University of Chinese Medicine, 2014.

[75] G. L. Sun, The Influence of Penetration Embedding Method on the [Master's, thesis], Henan University of Chinese Medicine, 2011.

[76] J. F. He, J. Yan, H. Y. Huang et al., "Intervention of acupuncture at stomach meridian acupoints for dio rat insulin resistant and hypothalamus IRS1 and tyrosine phosphorylation expression," Information on Traditional Chinese Medicine, vol. 30, no. 1, pp. 70-72, 2013.

[77] J. F. Qi, "Influence of electronic acupuncture on serum leptin and insulin of rats with simple obesity," Chinese Journal of Information on Traditional Chinese Medicine, vol. 14, no. 10, pp. 24-25, 2007.

[78] Q. H. Shao, Experimental Research on Electrical Auricularpoints for the Treatment of Simple Obesity [Master's, thesis], Nanjing University of Chinese Medicine, 2008.

[79] N. H. Huang, L. Hu, R. L. Cai et al., "Therapeutical effect on different gender obesity rat treated by electro-acupuncture the points of sanyinjiao and tianshu," Journal of Chengdu University of Traditional Chinese Medicine, vol. 30, no. 3, pp. 48-49, 2007.

[80] X. Liu, J.-F. He, Y.-T. Qu et al., "Electroacupuncture improves insulin resistance by reducing neuroprotein Y/agouti-related protein levels and inhibiting expression of protein tyrosine phosphatase $1 \mathrm{~b}$ in diet-induced obese rats," JAMS Journal of Acupuncture and Meridian Studies, vol. 9, no. 2, pp. 58-64, 2016.

[81] M. R. Gong, Y. Wang, J. Z. Jiang, and B. Xu, "Effect of different frequency electroacupuncture on expression of LR and IR in hypothalamus of obese rats," Lishizhen Medicine \& Materia Medica Research, vol. 22, no. 8, pp. 2031-2033, 2011.

[82] L. Han, Z. Jian-Bin, X. Chen et al., "Effects and mechanisms of auricular vagus nerve stimulation on high-fat-diet-induced obese rats," Nutrition Journal , vol. 31, no. 11-12, pp. 1416-1422, 2015.

[83] G. Yin, G. M. Shen, and Z. Q. Fang, "Influence of electroacupuncture on CRP and IL-6 expressions in adipose tissue of simple obesity rat," Chinese Journal of Gerontology, vol. 35, no. 6, pp. 1623-1624, 2015.

[84] G. Yin, G. M Shen, and Z. Q. Fang, "Effect of electroacupuncture at gastric back-shu and front-mu points on neuropeptide y in hypothalamus and serum in rats with simple obesity," Journal of Anhui Traditional Chinese Medical College, vol. 34, no. 2, pp. 49-52, 2015.

[85] C. Cao, Effects of Electro-Acupuncture on AMPK in Skeletal Muscle of Obese Rats [Master's thesis], Nanjing University of Chinese Medicine, 2014.

[86] J. Gao, C. L. Tang, R. J. Liu, X. L. Chen et al., "Effect of electroacupuncture in different intensities on endoplasmic reticulum stress in adipose tissues from high-fat diet-induced obese rats," Chinese Journal of Pathophysiology, vol. 35, no. 4, pp. 9899, 2013.

[87] Z. Yu, Y. B. Xia, C. H. Ju et al., "Research on the difference of electo-acupuncture at different pair points locating in abdomen and lower limb toward to adipo-hypothalamus cross-talk," Lishizhen Medicine \& Materia Medica Research, vol. 24, no. 1, pp. 238-241, 2013. 
[88] H. Z. Tang, L. Y. Zhi, S. J. Yan et al., "Influence of specific acupoint acupuncture therapy to serum angiogenesis factor of obese mouse models," Modernization of Traditional Chinese Medicine and Materia Medica-World Science and Technology, vol. 15, no. 6, pp. 1370-1374, 2013.

[89] D. S. Zhou, M. Li, and H. Hu, "The expression of MMP-9 and encephaledema after cerebral infarction," Journal of Traditional Chinese Medicine, vol. 30, no. 12, pp. 2676-2678, 2012.

[90] Y. Luo, B. Li, and G. B. Huang, "Weight-reducing Effect of Acupuncture in Obesity Rats," Shanghai Journal of Acupuncture Moxibustion, vol. 31, no. 7, pp. 528-529, 2012.

[91] Q. Yuan, R. Jin, and B. W. Ouyang, "Studies of "Fei Shan Zen" in treatment model of rats simply obesity," Acta Chinese Medicine \& Pharmacology, vol. 31, no. 6, pp. 34-36, 2003.

[92] X. D. Li, Establishment of diet induced obesity model in rats and study on the mechanism of obesity treated by electroacupuncture [Master's thesis], Tianjin Medical University, 2003.

[93] N. Tian, Study on Orexigenic Peptides mechanism of Obesity Treated by Electroacupuncture [Master's thesis], Dalian Medical University, 2005.

[94] Z. C. Zhang and Z. Sun, "Morphological observation on acupuncture treatment effect on fat cell of dietinduced obesity rat," Chinese Journal of Behavioral Medical Science, vol. 15, no. 7, pp. 580-582, 2006.

[95] S. F. Yang and Y. W. Wang, "Effects of acupoint catgutembedding on NO, and NOS in striatum of rats with simple obesity," ZheJiang Journal of Traditional Chinese Medicine, vol. 43, no. 7, pp. 380-381, 2008.

[96] M.-R. Gong, B. Xu, Z. Mao, and Q.-H. Shao, "Effect of acupuncture on the expression of leptin receptor in hypothalamus in obesity rats: Determination by SYBR green realtime quantitative polymerase chain reaction," Journal of Clinical Rehabilitative Tissue Engineering Research, vol. 12, no. 46, pp. 9105-9108, 2008.

[97] H. Yang, Y. Li, L. Cheng et al., "Effects of electroacupuncture and diet on blood lipid and adiponectin in nutritional obese rats," Acta Universitatis Traditionis Medicalis Sinensis Pharmacologiaeque Shanghai, vol. 21, no. 5, pp. 65-67, 2007.

[98] L. Gao, X.-J. Kong, and X. Shi, "Effects of electroacupuncture and acupoint catgut-embedding on mRNA expression of lipid metabolism gene PPAR-gamma and related lipase of rats with simple obesity," Zhongguo Zhen Jiu = Chinese Acupuncture \& Moxibustion, vol. 31, no. 6, pp. 535-538, 2011.

[99] M. Yu, X. Q. Xiao, C. L. Tang et al., "Effect of different intensities of electroacupuncture on expression of monocyte chemoattractant protein-1 and TNF-alpha in adipose tissue in obesity rats," Acupuncture Research, vol. 36, no. 2, pp. 79-84, 2011.

[100] Z. L. Liu, C. L. Tang, M. Yu et al., "Effect of different intensities of electroacupuncture on the expression of C-reactive protein and interleukin-6 in adipose tissues of simple obesity rats," Journal of Traditional Chinese Medicine, vol. 52, no. 8, pp. 693-696, 2011.

[101] B. Xu, J. H. Yuan, Z. Liu et al., "The Research in the difference of Effects of electroacupuncture and sibutramine on adipocyte products in obesity rats," in Proceeding of the China Association and Literature Committee of Acupuncture-Moxibustion Conference Proceedings, 2007.

[102] D.-R. Tian, X.-D. Li, F. Wang et al., "Up-regulation of the expression of cocaine and amphetamine-regulated transcript peptide by electroacupuncture in the arcuate nucleus of dietinduced obese rats," Neuroscience Letters, vol. 383, no. 1-2, pp. 17-21, 2005.
[103] L. H. Wang, Experimental Research of Embedded Thread Therapy for Simple Obesity [Master's, thesis], Guangzhou University of Chinese Medicine, 2008.

[104] C. Yu, Experimental Research on Different Acupuncture Point Groups for the Influence of Related Parameters of Simple Obesity Rats [Master's, thesis], Beijing University of Chinese Medicine, 2008.

[105] Y. A. Chen, Effect of Acupuncture and Moxibustion at Guanyuan (CV 4) on the Intervention of Nonestesterified Fatty Acid in Obesity Rats [Master's, thesis], Beijing University of Chinese Medicine, 2012.

[106] Y. J. Zhang, The Experimental Study on Treatment of Simple Obesity with Electroacupuncture at Zusanli [Master's, thesis], Jilin University, 2012.

[107] X. J. Luo, Neural Mechanism Study for Obese Adolescence: Evaluated with MR-Neuroimages and Laboratory Animals [Master's thesis], Peking Union Medical College, 2013.

[108] X. Y. Tao, The Animal Experiment and Clinical Research on Fat 3needIeTherapy for Simple Obesity [Master's, thesis], Guangzhou University of Chinese Medicine, 2013.

[109] J. Lin, Effect of Electric Acupuncture Points at Different Positions of the Weight Difference in Mechanism and Experimental Study [Master's, thesis], Nanjing University of Chinese Medicine, 2012.

[110] L. F. Hong, Research on the Mechanism of Weight Loss in DIO Rats by Acupuncture [Master's, thesis], Beijing University of Chinese Medicine, 2003.

[111] X. M. Zhang, Experimental Study of Acupuncture and Embedded Thread Therapy on Obesity [Master's, thesis], Guangzhou University of Chinese Medicine, 2006.

[112] Y. J. Fu, Y. P. Bo, and Z. J. Lun, "Effect of acupuncture of stomach-meridian acupoints on brown adipocytes in extra weight rats," Journal of Guangzhou University of Traditional Chinese Medicine, vol. 22, no. 2, pp. 127-129, 2005.

[113] T. Shiraishi, M. Onoe, T. Kojima, Y. Sameshima, and T. Kageyama, "Effects of auricular stimulation on feeding-related hypothalamic neuronal activity in normal and obese rats," Brain Research Bulletin, vol. 36, no. 2, pp. 141-148, 1995.

[114] R. H. Yan, The Study of Clinical Effect and Molecular Mechanism in Treating Simple Obesity with Catgut Implantation at Acupoint [Master's, thesis], Dalian Medical University, 2013.

[115] B. Ji, J. Hu, and S. Ma, "Effects of electroacupuncture Zusanli (ST36) on food intake and expression of POMC and TRPV1 through afferents-medulla pathway in obese prone rats," Peptides, vol. 40, pp. 188-194, 2013.

[116] W. Shen, Y. Wang, S.-F. Lu et al., "Acupuncture promotes white adipose tissue browning by inducing UCP1 expression on DIO mice," BMC Complementary and Alternative Medicine, vol. 14, no. 1, article 501, 2014.

[117] A. L. Wu, The Clinical Evaluation and Laboratory Studies with Catgut Embedding Treatment for Obesity [Master's, thesis], Guangzhou University of Chinese Medicine, 2009.

[118] A. Eduardo, Simple Obesity Treatment in Animals Model Using Chinese Herbs and Acupuncture [Master's, thesis], Tianjin Medical University, 2003.

[119] J. C. Leng, Y. Luo, H. M. Zhang et al., "Effect of electroacupuncture on expression of DNA methyltransferase 1 in hypothalamus of obese rats," Hunan Journal of Traditional Chinese Medicine, vol. 31, no. 11, pp. 156-158, 2015.

[120] M. Deng, Y. N. Sun, and M. R. Gong, "The effect of catgut implantation at acupoint on obesity rats," Liaoning Journal of Traditional Chinese Medicine, vol. 44, no. 2, pp. 386-388, 2016. 
[121] H. Sun, N. N. Yang, X. F. Huang et al., "Role of brown adipose tissue characteristic genes in resisting obesity," Chinese Journal of Clinical Gastroenterology, vol. 25, no. 1, pp. 3-9, 2013.

[122] H. Y. Li, H. Z. Tang, Y. Pang et al., "Research on the effects of specific points on losing weight," Lishizhen Medicine and Materia Medica Research, vol. 23, no. 6, pp. 1466-1467, 2012.

[123] Q. L. Yao, M. W. Li, P. Abudureyimu et al., "Effect of acupuncture on obesity parameters and morphology of fat tissue in obese rats," Journal of Xinjiang Medical University, vol. 28, no. 7, pp. 608-610, 2005 (Chinese).

[124] J. Su, Z. C. Liu, and M. Zhao, "Research on the effect of satiety center in the reduction of weight by electropuncture," Shanghai Journal of Acuuncture \& Moxibustion, vol. 18, no. 6, pp. 30-31, 1999.

[125] B. L. Liu, L. P. Wang, F. J. Chen et al., "Experimental research for the catgut implantation at four body acupoints treatment of simple obesity rats," Chinese practical Chinese and western medicine, pp. 2091-2093, 2007.

[126] Z. Q. Fang, G. Yin, G. S. Xu et al., "Changes of electrogastroenterography in rats with simple obesity and modulated effect of acupuncture on them," Chinese Journal of Traditional Medical Science and Technology, vol. 12, no. 4, pp. 203-204, 2005.

[127] C.-K. Wen and T.-Y. Lee, "Electroacupuncture decreases the leukocyte infiltration to white adipose tissue and attenuates inflammatory response in high fat diet-induced obesity rats," Evidence-Based Complementary and Alternative Medicine, vol. 2014, Article ID 473978, 11 pages, 2014.

[128] W. Wang, The Experimental Study on Acupuncture Treatment of Simple Obesity in Rats [Master's thesis], Jilin University, 2009.

[129] J.-J. Ge, S.-J. Wang, L.-H. Sun, Q. Li, and H.-L. Xiao, "Effects of electroacupuncture of different frequencies on slimming effect in the rat of experimental obesity," Zhongguo Zhen Jiu = Chinese Acupuncture \& Moxibustion, vol. 27, no. 8, pp. 598-600, 2007.

[130] L. Zheng, Q. Y. Xu, and Y. Zhou, "The research on the effects of electroacupuncture of losing weight on ob/ob mice," AsiaPacific Traditional Medicine, vol. 11, no. 8, pp. 12-13, 2015.

[131] L. Zheng, M. Y. Fang, J. S. Zhou et al., "Effect of aerobic exercise and acupuncture on abdominal fat and other indexes of rats," China Sport Science \& Technology, vol. 42, no. 2, pp. 122-124, 2006.

[132] W. Lihua, L. Jia, H. Wei et al., "Effect of electroacupuncture on the expression of PGC- $1 \alpha$ and UCP- 1 in the brown adipose tissue of obese rats," World Journal of Acupuncture - Moxibustion, vol. 28, no. 2, pp. 97-102, 2018.

[133] Y. C. Si, W. N, J. Y. Miao et al., "Effects of acupuncture on $\mathrm{T}$ lymphocyte subsets in thymus, spleen and intestinal peyer' patches of obesity mice," Lishizhen Medicine and Materia Medica Research, vol. 29, no. 8, pp. 2043-2045, 2018.

[134] Y. X. Tang, J. Z. Meng, Y. Y. Ding et al., "Effect of electroacupuncture on adenosine receptor expression in white adipose tissue of dietinduced obese mice," Acupuncture Research, vol. 42, no. 1, pp. 39-44, 2017.

[135] J. J. Wu, Y. W. Zhu, and N. Y. Sun, "Effects of preventive acupuncture at the fenglong acupoint on obesity prevention and PPAR- $\gamma$ expression level in rats fed a high-fat diet," Journal of Chinese General Practice, vol. 22, no. 6, pp. 707-711, 2019.

[136] Y. C. Si, W. N. Miao, G. X. Wu et al., "Effects on the regulatory function of intestinal mucosal TLR1/TLR2 gene in nutritional obese mice by acupuncture," Journal of Traditional Chinese Medicine, vol. 34, no. 3, pp. 1166-1169, 2019.
[137] J. P. Yao, Y. Li, L. Zhang et al., "Effect of electroacupuncture on hypothalamic autophagy-related protein 7 expression in diet-induced obesity rats," Journal of Guangzhou University of Traditional Chinese Medicine, vol. 36, no. 4, pp. 545-548, 2019.

[138] S. Y. Zhang, X. Hu, H. T. Tang et al., "Effect of acupuncture on macrophage polarization of white adipose tissue in obese mice induced by high-fat diet," Chinese Acupuncture \& Moxibustion, vol. 37, no. 11, pp. 1205-1211, 2017.

[139] N. Y. Yang, Q. Shu, L. Chen et al., "Mechanism of electroacupuncture mediated hepatic foxol in regulating lipid metabolism of obese rats," Chinese Archives of Traditional Chinese Medicine, vol. 36, no. 9, pp. 2129-2133, 2018.

[140] X. Y. Li, "Effect of different levels catgut embedding at acupoints on obesity and glucose and lipid metabolism in rats with simple obesity," Professional committee of experimental medicine, Chinese academy of integrated traditional and western medicine," Collection of Papers of the 14th China Symposium on Experimental Medicine of Integrated Traditional Chinese and Western Medicine, 2017.

[141] X. L. Fan, Study on the Intestinal Microflora of Obese Rats with Acupuncture Treatment by $16 S r$ DNA Technology [Master's, thesis], Guangxi University of Chinese Medicine, 2018.

[142] L. D. Lin, Proteomics Analysis Based on I TRAQ Technology of Acupuncture on the Hypothalamus of Diet-Induced Obesity Rats [Master's, thesis], Guangxi University of Chinese Medicine, 2018.

[143] Y.Z. He, The Clinical Effects of Cognitive Dysfunction of Cerebral Palsy in Adjusting Shen and Expediting Du Therapy Combined with Cognitive Function Training [Master's, thesis], Guangxi University of Chinese Medicine, 2018.

[144] K. Li, H. He, M. Q. Hu et al., "Mechanism of electroacupuncture for reducing diet-induced obesity rat weight through hypothalamus TSC1-mTOR signal pathway," Chinese Journal of Integrated Traditional and Western Medicine, vol. 36, no. 7, pp. 875-878, 2016.

[145] H. J. Zhou, H. Wang, Q. Shu et al., "Dietary restriction on hypothalamo-pituitary-adrenal axis and the metabolism of glucolipid in obesity rats," Modern Preventive Medicine, vol. 43, no. 16, pp. 2926-2929, 2016.

[146] Y. H. Wei, Effect of Electro-acupuncture at, Fenglong, Point on Liver Lipid Metabolism in Obese Rats and the Mechanism of Response to Related Molecular Networks [Master's, thesis], Nanjing University of Chinese Medicine, 2018.

[147] L. S. Wang, Y. Li, L. L. Zhong et al., "Effect of electroacupuncture on obesity index and fat cell morphology of obese rats," Journal of Clinical Acupuncture and Moxibustion, vol. 32, no. 10, pp. 89-92, 2016.

[148] S. S. Xu, M. Deng, Y. N. Sun et al., "Effects of electroacupuncture on weight reduction and inflammatory factor in adipose tissues of obese mice," Liaoning Journal of Traditional Chinese Medicine, vol. 44, no. 2, pp. 386-388, 2017. 


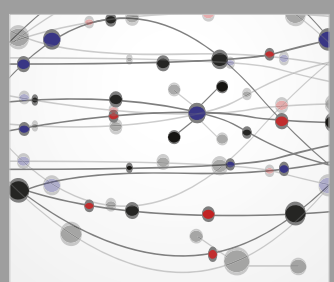

The Scientific World Journal
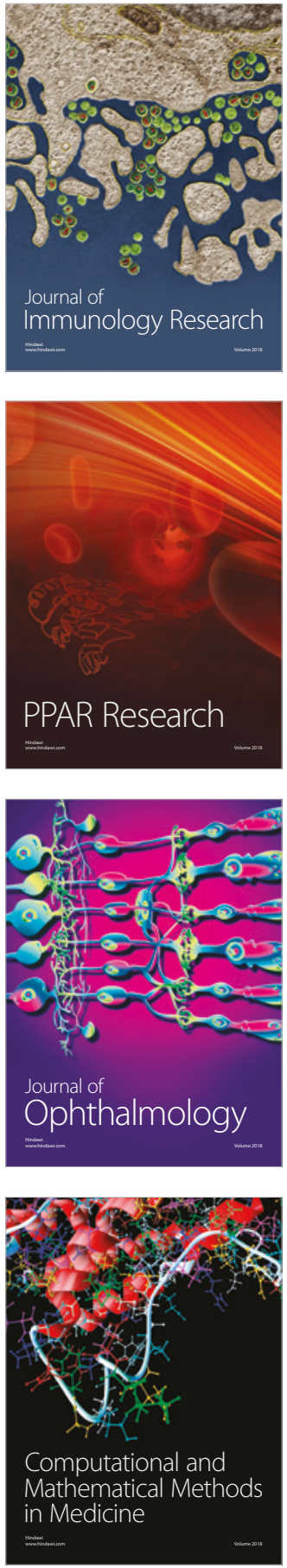

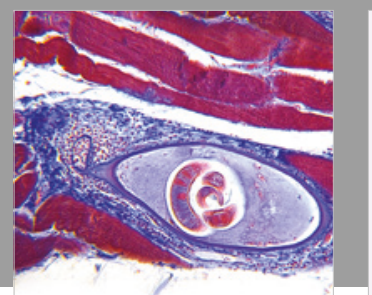

Gastroenterology Research and Practice

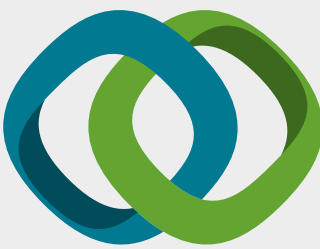

\section{Hindawi}

Submit your manuscripts at

www.hindawi.com
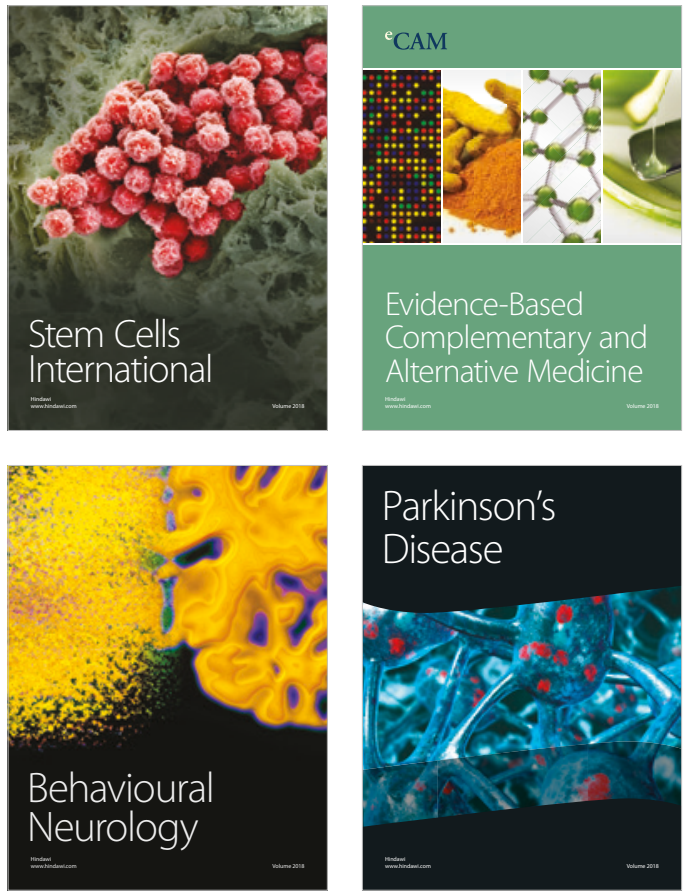

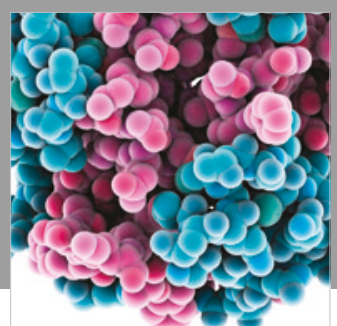

ournal of

Diabetes Research

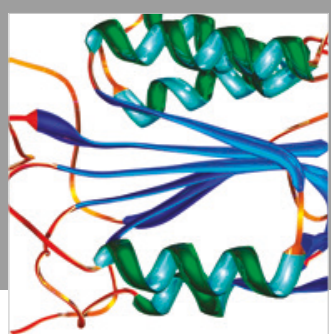

Disease Markers
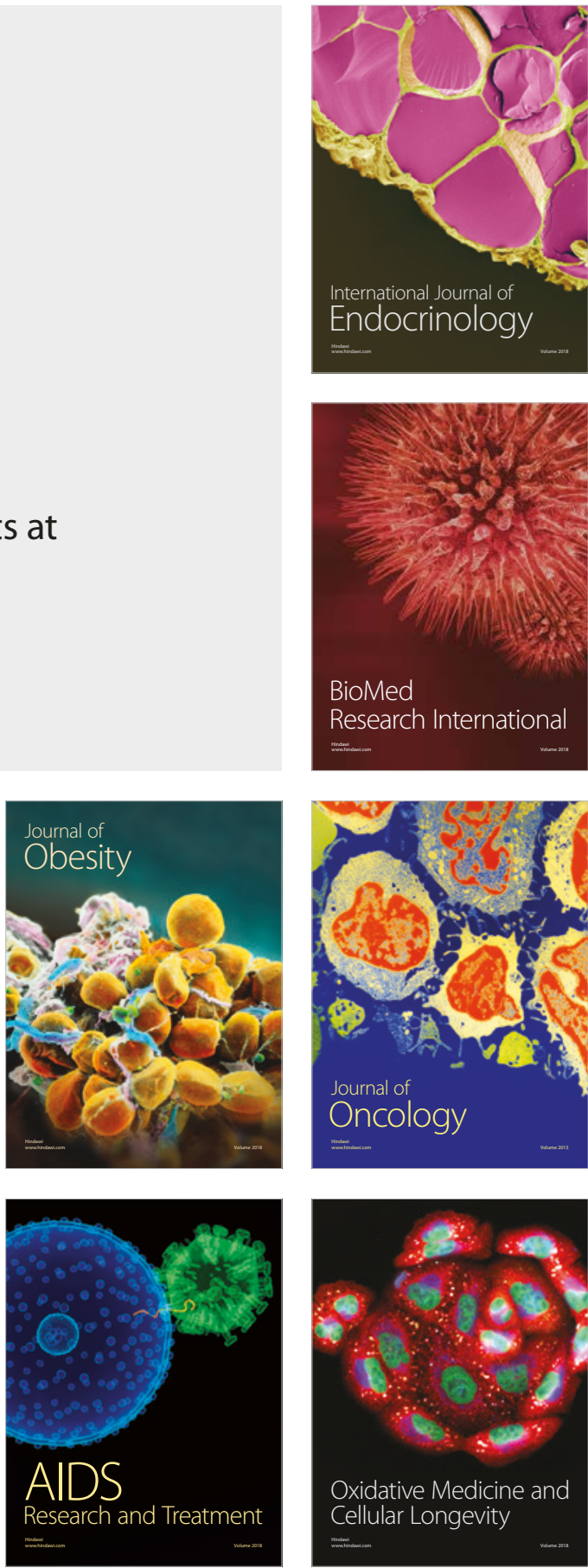